TRANSACTIONS OF THE

AMERICAN MATHEMATICAL SOCIETY

Volume 365, Number 9, September 2013, Pages 4963-4986

S 0002-9947(2013)05793-5

Article electronically published on April 2, 2013

\title{
LOWER BOUNDS OF GROWTH OF HOPF ALGEBRAS
}

\author{
D.-G. WANG, J. J. ZHANG, AND G. ZHUANG
}

Abstract. Some lower bounds of GK-dimension of Hopf algebras are given.

\section{INTRODUCTION}

A seminal result of Gromov states that a finitely generated group has polynomial growth or, equivalently, the associated group algebra has finite Gelfand-Kirillov dimension if and only if it has a nilpotent subgroup of finite index Gr. Group algebras form a special class of cocommutative Hopf algebras. It is natural to ask

Question 0.1. What are necessary and sufficient conditions on a finitely generated Hopf algebra $H$ such that its Gelfand-Kirillov dimension is finite?

Let $k$ be a base field and everything be over $k$. Assume that, for simplicity, $k$ is algebraically closed of characteristic zero. It is clear that an affine (i.e., finitely generated) commutative Hopf algebra has a finite GK-dimension (short for GelfandKirillov dimension) which equals its Krull dimension. If $H$ is cocommutative, by a classification result [Mo, Corollary 5.6.4 and Theorem 5.6.5], it is isomorphic to a smash product $U(\mathfrak{g}) \# k G$ for some group $G$ and some Lie algebra $\mathfrak{g}$. Consequently,

$$
\operatorname{GKdim} H=\operatorname{GKdim} k G+\operatorname{dim} \mathfrak{g},
$$

which solves Question 0.1 in terms of conditions on $G$ and $\mathfrak{g}$. Question 0.1 is also answered for several classes of noncommutative and noncocommutative Hopf algebras, including quantum groups $U_{q}(\mathfrak{g})$ and $\mathcal{O}_{q}(G)$; see BG2, GZ1. The present paper attempts to study Question 0.1 for a larger class of noncommutative and noncocommutative Hopf algebras by providing three lower bounds of GK-dimension in terms of certain invariants of skew primitive elements.

Let $H$ be a Hopf algebra over $k$. A nonzero element $y \in H$ is called $(1, g)$ primitive (or generally skew primitive) if $\Delta(y)=y \otimes 1+g \otimes y$, and such a $g$ is called the weight of $y$ and denoted by $\mu(y)$. Let $G(H)$ denote the group of group-like elements in $H$ and let $C_{0}=k G(H)$. Here is the first lower bound theorem.

Theorem 0.2 (First lower bound theorem). Let $D \supseteq C_{0}$ be a Hopf subalgebra of $H$. Let $\left\{y_{i}\right\}_{i=1}^{w}$ be a set of skew primitive elements such that

(a) $\left\{y_{i}\right\}_{i=1}^{w}$ is linearly independent in $H / D$,

(b) for all $i \leq j, y_{i} \mu\left(y_{j}\right)=\lambda_{i j} \mu\left(y_{j}\right) y_{i}$ for some $\lambda_{i j} \in k^{\times}$,

(c) for each $i, \lambda_{i i}$ is either 1 or not a root of unity.

Then GKdim $H \geq \operatorname{GKdim} D+w$.

Received by the editors September 13, 2010 and, in revised form, October 26, 2011, November 22, 2011 and January 17, 2012.

2010 Mathematics Subject Classification. Primary 16P90, 16T05; Secondary 16T20.

Key words and phrases. Hopf algebra, Gelfand-Kirillov dimension, skew primitive, pointed. 
In general $\lambda_{i j}$ in condition (b) may not exist. If that is the case, we have other ways of obtaining lower bounds.

Let $W$ denote the set of weights $\mu(y)$ for all skew primitive elements $y \notin C_{0}$ and let $W_{\sqrt{ }}$ be the subset of $W$ consisting of weights $\mu(y)$ for all $y$ such that $y^{n}$ is also a skew primitive for some $n>1$. (Note that in this paper the term "skew primitive" means "( $1, g)$-primitive".) For any subset $\Phi \subset G(H)$, the subgroup of $G(H)$ generated by $\Phi$ is denoted by $\langle\Phi\rangle$. Here is the second lower bound theorem.

Theorem 0.3 (Second lower bound theorem). Suppose $\left\langle W \backslash W_{\sqrt{ }}\right\rangle$ is abelian. Then

$$
\operatorname{GKdim} H \geq \operatorname{GKdim} C_{0}+\#\left(W \backslash W_{\sqrt{ }}\right) .
$$

There are examples such that $W=W_{\sqrt{ }}$ and $\operatorname{GKdim} H=\operatorname{GKdim} C_{0}$, but $\#\left(W_{\sqrt{ }}\right)$ is arbitrarily large [Example 2.7. Therefore $W_{\sqrt{ }}$ has to be removed from $W$ when we estimate the GK-dimension of $H$.

Let $y$ be a skew primitive element not in $C_{0}$. If

$$
\mu(y)^{-1} y \mu(y)-c y \in C_{0}
$$

for some $c \in k^{\times}$, then $c$ is called the commutator of $y$ (with its weight) and is denoted by $\gamma(y)$. By Lemma 1.6, (I0.3.2) is equivalent to

$$
\mu(y)^{-1} y \mu(y)-c y=\tau(\mu(y)-1)
$$

for some $\tau \in k$. Define $\Gamma$ to be the set of $\gamma(y)$ for all skew primitive elements $y \notin C_{0}$ such that $\gamma(y)$ exists, and let $\Gamma_{\sqrt{ }}$ be the subset of $\Gamma$ consisting of those $\gamma(y)$ which are roots of unity but not 1 . If $\gamma(y)$ exists, the pair $(\mu(y), \gamma(y))$ is denoted by $\omega(y)$ and is called the weight commutator of $y$. When (10.3.3) holds and if $c \neq 1, y$ can be replaced by $z:=y+(c-1)^{-1} \tau(\mu(y)-1)$, which is a skew primitive element with $\omega(z)=\omega(y)$ and satisfies the equation $\mu(z)^{-1} z \mu(z)-\gamma(z) z=0$.

Define $\Omega$ to be the set of $\omega(y)$ for all skew primitive elements $y \notin C_{0}$ such that $\omega(y)$ exists, and let $\Omega_{\sqrt{ }}$ be the subset of $\Omega$ consisting of those $\omega(y)$ in which $\gamma(y)$ is a root of unity but not 1 . Theorem 0.3 can be improved a little under the same hypothesis:

$$
\operatorname{GKdim} H \geq \operatorname{GKdim} C_{0}+\#\left(\Omega \backslash \Omega_{\sqrt{ }}\right) .
$$

Let $y$ be a skew primitive element not in $C_{0}$ with $g=\mu(y)$. Let $T_{g^{-1}}$ be the inverse conjugation by $g$, namely, $T_{g^{-1}}: a \rightarrow g^{-1} a g$. A scalar $c$ is called a commutator of $y$ of level $n$ if $n$ is the least nonnegative integer such that

$$
\left(T_{g^{-1}}-c I d_{H}\right)^{n}(y) \in C_{0} .
$$

In this case we also write $\gamma(y)=c$. Let $Z$ denote the space spanned by the identity element 1 and all skew primitive elements of $H$, and let $Y_{\sqrt{ }}$ denote the subspace of $Z$ spanned by those $y$ with commutator of finite level and with $\gamma(y)$ being a root of unity but not 1 . Here is the third lower bound theorem. Let $W_{\times}$be the subset of $W$ consisting of weights $\mu(y)$ such that the commutator of $y$ (as defined in (I0.3.4) exists and is either 1 or not a root of unity. Note that $W \backslash W_{\sqrt{ }} \subseteq W_{\times}$, and these are often equal [Remark 3.9].

Theorem 0.4 (Third lower bound theorem). Suppose $\left\langle W_{\times}\right\rangle$is abelian. Then

$$
\operatorname{GKdim} H \geq \operatorname{GKdim} C_{0}+\operatorname{dim} Z /\left(C_{0}+Y_{\sqrt{ }}\right) .
$$


When $H$ is cocommutative, equality holds in Theorem 0.4 see (I0.1.1). There are examples such that $Z=Y_{\sqrt{ }}+C_{0}$ and $\operatorname{GKdim} H=\operatorname{GKdim} C_{0}$, but $\operatorname{dim} Y_{\sqrt{ }}$ is arbitrarily large [Examples 2.7 and 3.13. Therefore it is sensible to consider the quotient space $Z /\left(C_{0}+Y_{\sqrt{ }}\right)$ in the above theorem. This is analogous to removing $W_{\sqrt{ }}$ in Theorem 0.3 .

If $\left\langle W_{\times}\right\rangle$is abelian, Theorem 0.4 is a generalization of Theorem 0.3 [Lemma 3.12 . After some analysis, Theorem 0.2 (when $D=C_{0}$ ) can be viewed as a consequence of Theorem 0.4 These lower bounds provide some evidence that the GK-dimension of $H$ is related to some combinatorial data coming from the skew primitive elements when $H$ is pointed.

The proof of these lower bounds is based on a version of the Poincaré-BirkhoffWitt (PBW) theorem [Theorem [1.5(b)] which states that under some hypotheses the set of monomials generated by skew primitive elements is linearly independent (over the Hopf subalgebra $C_{0}$ ). Restricted to the universal enveloping algebra of a finite dimensional Lie algebra, Theorem 1.5 implies the original PBW theorem. Theorem [1.5] is in a similar spirit to Kharchenko's quantum analog of the PBW theorem [Kh]. One of the hypotheses in Theorem 1.5] is (I1.2.3) which assumes essentially that the action of the group generated by weights on the space generated by skew primitive elements is locally finite. When GKdim $H$ is finite, this is a reasonable hypothesis indicated by a result of the third-named author $\mathrm{Zhu}$, Theorem 1.2] (see also Lemma 2.5).

In general we are far from answering Question 0.1. There are many unsolved questions concerning the growth of Hopf algebras. The hypotheses in Theorems 0.3 and 0.4 could be superfluous, but we don't know how to remove them at this moment. When $\langle W\rangle$ is nonabelian, a possible better lower bound could be obtained by replacing $\#\left(W \backslash W_{\sqrt{ }}\right)$ in Theorem 0.3 by GKdim $k\langle W\rangle$; see Lemma 2.6 (b) for details. It is expected that these lower bounds can (or should) be improved and that possible upper bounds should be found once finer invariants are introduced. The ultimate goal is to find a formula for the GK-dimension of a Hopf algebra which is analogous to Bass' theorem [KL, Theorem 11.14] in the group algebra case, and then eventually to solve Question 0.1 .

There are further connections between the growth of Hopf algebras and $W$ and other invariants defined by skew primitive elements. Let rank denote the torsionfree rank of an abelian group.

Proposition 0.5. Suppose $\langle W\rangle$ is abelian and torsionfree. If $\operatorname{rank}\langle\Gamma\rangle>\operatorname{rank}\langle W\rangle=$ 1, then $H$ has exponential growth.

Note that $\operatorname{rank}\left\langle\Gamma \backslash \Gamma_{\sqrt{ }}\right\rangle=\operatorname{rank}\langle\Gamma\rangle$ since elements in $\Gamma_{\sqrt{ }}$ have finite order. The rank of $\langle W\rangle$ and $\langle\Gamma\rangle$ should be related when GKdim $H$ is finite.

Question 0.6. Suppose $\operatorname{rank}\langle\Gamma\rangle>\operatorname{rank}\langle W\rangle$. Does $H$ then have exponential growth?

Quite a few families of Hopf algebras of finite GK-dimension have been analyzed extensively by several authors [AA, AS1, AS2, Br1, Br2, BG1, BG2, BZ, GZ1, GZ2, LWZ, WuZ1, WuZ2, Zhu] during the last few years. But the classification of such Hopf algebras is far from complete. These lower bounds are useful for studying pointed Hopf algebras of low GK-dimension. For example, if GKdim $H=2$, then

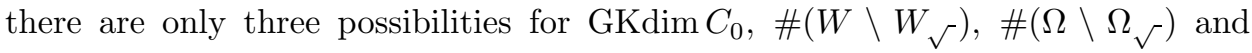


$\operatorname{dim} Z /\left(C_{0}+Y_{\sqrt{ }}\right)$. This is one of the initial steps in our ongoing project of classifying pointed Hopf algebra domains of GK-dimension two and three.

Definitions and basic properties of GK-dimension can be found in the first three chapters of $[\mathrm{KL}$. Our reference book for Hopf algebras is $[\mathrm{Mo}]$.

\section{FiRst LOWER BOUND THEOREM}

In this section we prove Theorem 0.2 We need some lemmas.

Lemma 1.1. Let $D$ be a Hopf subalgebra of $H$ and $0 \neq F \in H$. Suppose that

(a) $L$ is a subcoalgebra of $H$ containing $D$,

(b) $L$ is a left $D$-module via the multiplication, and

(c) there are nonzero-divisors (regular elements) $h, g \in L$ such that $\Delta(F)-F \otimes$ $h-g \otimes F \in L \otimes L$.

Define $V=\{a \in D \mid a F \in L\}$. Then $V$ is either 0 or $D$.

Proof. Suppose $V$ is nonzero and let $a$ be a nonzero element in $V$. Let $C$ be the subcoalgebra of $D$ generated by $a$. There is a $k$-linear basis

$$
\left\{a_{1}, \cdots, a_{v}, a_{v+1}, \cdots, a_{w}\right\}
$$

of $C$ such that $C \cap V$ is spanned by $\left\{a_{1}, \cdots, a_{v}\right\}$. This means that $a_{i} F \in L$ for all $i \leq v$ and that any nontrivial linear combination of $\left\{a_{v+1} F, \cdots, a_{w} F\right\}$ is not in $L$. Write $\Delta(a)=\sum_{1 \leq i, j \leq w} \xi_{i j} a_{i} \otimes a_{j}$ for some $\xi_{i j} \in k$.

For simplicity, we use the symbol $l d t_{1}$ for any element in $L$ and use $l d t_{2}$ for any element in $L \otimes L$. By the definition of $V$, we have $a F+l d t_{1}=0$ for some $l d t_{1} \in L$, and hence

$$
\begin{aligned}
0 & =\Delta\left(a F+l d t_{1}\right)=\Delta(a) \Delta(F)+\Delta\left(l d t_{1}\right) \\
& =\left(\sum_{i, j} \xi_{i j} a_{i} \otimes a_{j}\right)\left(F \otimes h+g \otimes F+l d t_{2}\right)+l d t_{2} \\
& =\left(\sum_{i, j} \xi_{i j} a_{i} \otimes a_{j}\right)(F \otimes h)+\left(\sum_{i, j} \xi_{i j} a_{i} \otimes a_{j}\right)(g \otimes F)+l d t_{2} \\
& =\left(\sum_{\substack{i>v \\
\text { all } j}} \xi_{i j} a_{i} \otimes a_{j}\right)(F \otimes h)+\left(\sum_{\substack{j>v \\
\text { all } i}} \xi_{i j} a_{i} \otimes a_{j}\right)(g \otimes F)+l d t_{2},
\end{aligned}
$$

where the last equation uses the fact $a_{i} F \in L$ for all $i \leq v$. The above equation implies that

$$
\left(\sum_{\substack{j>v \\ \text { all } i}} \xi_{i j} a_{i} \otimes a_{j}\right)(g \otimes F)=-\left(\sum_{\substack{i>v \\ \text { all } j}} \xi_{i j} a_{i} \otimes a_{j}\right)(F \otimes h)+l d t_{2} \in H \otimes L
$$

or, equivalently, $\sum_{i=1}^{w}\left(a_{i} g\right) \otimes\left(\sum_{j>v} \xi_{i j} a_{j} F\right) \in H \otimes L$. Since $\left\{a_{i} g\right\}_{i=1}^{w}$ is linearly independent, we have $\sum_{j>v} \xi_{i j} a_{j} F \in L$ for all $i$. By the definition of $\left\{a_{v+1}, \cdots, a_{w}\right\}$, we obtain that $\xi_{i j}=0$ for all $j>v$. Similarly, $\xi_{i j}=0$ for all $i>v$. Thus $\Delta(a) \in V \otimes V$, and hence $V$ is a subcoalgebra of $D$. Since $V$ is a subcoalgebra, there is an element $v \in V$ such that $\epsilon(v)=1$. Then

$$
1 F=\epsilon(v) F=\sum S\left(v_{1}\right) v_{2} F \in L
$$

since $v_{2} \in V$ and $S\left(v_{1}\right) \in D$. This shows that $1 \in V$. Since $V$ is a left ideal of $D$, $V=D$. 
Remark 1.2. Recall that $\left(\mathbb{N}^{v},+\right)$, for every $v \geq 1$, is a linearly ordered semigroup with respect to the following ordering. Define $\left(c_{1}, \cdots, c_{v}\right)<\left(d_{1}, \cdots, d_{v}\right)$ if either $\sum_{i=1}^{v} c_{i}<\sum_{i=1}^{v} d_{i}$ or $\sum_{i=1}^{v} c_{i}=\sum_{i=1}^{v} d_{i}$ and there is a $p<v$ such that $c_{i}=d_{i}$ for all $i \leq p$ and $c_{p+1}<d_{p+1}$.

Let $T:=k\left\langle\left\{x_{j}\right\}_{j \in J}\right\rangle$ be the free algebra generated by $\left\{x_{j}\right\}_{j \in J}$. Given any family $\left(f_{j}\right)_{j \in J}$ where $f_{j} \in \mathbb{N}^{v}$, we can define an $\mathbb{N}^{v}$-graded structure on $T$ by setting $\operatorname{deg} x_{j}=f_{j}$ for all $j \in J$. Then $T=\bigoplus_{w \in \mathbb{N}^{v}} T_{w}$. Since $\mathbb{N}^{v}$ is linearly ordered, $T$ has a canonical $\mathbb{N}^{v}$-filtration defined by $F_{w}(T)=\sum_{w^{\prime} \leq w} T_{w^{\prime}}$. Let $B$ be any factor ring of $T$. The $\mathbb{N}^{v}$-filtration on $T$ induces a unique $\mathbb{N}^{v}$-filtration on $B$, denoted by $\left\{F_{w}(B) \mid w \in \mathbb{N}^{v}\right\}$. We say that an element $x \in B$ has filtered multi-degree

$$
\operatorname{deg} x:=w=\left(d_{1}, \cdots, d_{v}\right)
$$

and filtered total-degree $d=\sum d_{i}$ if $x \in F_{w}(B) \backslash \sum_{w^{\prime}<w} F_{w^{\prime}}(B)$. Note that the filtered total-degree induces an $\mathbb{N}$-filtration on $B$.

In applications, we usually start with an algebra $A$ generated by $\left\{y_{1}, \cdots, y_{v}\right\}$ and $G=\left\{g_{i}\right\}_{i \in I}$ for some index set $I$. By the discussion in the previous paragraph, we can define two filtrations (and corresponding filtered degrees) on $A$ such that if $f=y_{i_{1}} y_{i_{2}} \cdots y_{i_{s}} \in A$, then the filtered total $y$-degree of $f$ is at most $s$ and the filtered multi-y-degree of $f$ is at most $\left(n_{1}, \cdots, n_{v}\right)$, where $n_{i}$ is the number of $y_{i}$ appearing in $F$, and if $g \in G$, the filtered total- $y$-degree and the filtered multi- $y$ degree of $g$ are both 0 .

These two filtrations can be extended to the tensor product $A \otimes A$, namely, $F_{w}(A \otimes A):=\sum_{w^{\prime}+w^{\prime \prime}<w} F_{w^{\prime}}(A) \otimes F_{w^{\prime \prime}}(A)$ for all $w \in \mathbb{N}^{v}($ or $w \in \mathbb{N}$ ). For simplicity, the words "filtered" and "filtration" might be omitted below.

Assume that $S:=\left\{y_{i}\right\}_{i \in I}$ is a set of skew primitive elements of $H$ where $I$ is either $\mathbb{N}$ or $\{1, \cdots, v\}$ for some positive integer $v$. Suppose that $D$ is a Hopf subalgebra of $H$ and that

(I1.2.1) $g_{i}:=\mu\left(y_{i}\right) \in D$ for all $i \in I$,

(I1.2.2) $S$ is linearly independent in the space $H / D$,

(I1.2.3) for each pair $i \leq j, y_{i} g_{j}=\lambda_{i j} g_{j} y_{i}+b_{i j}$ for some $\lambda_{i j} \in k^{\times}$and $b_{i j} \in D$, and there is a subalgebra $A \subset D$ containing all $b_{i j}$ such that $y_{i} A \subset A y_{i}+A$ and $g_{i} A \subset A g_{i}+A$ for all $i$.

In most of the applications $D$ is the coradical $C_{0}$ of $H$ and the commutators of the $y_{i}$ exist. When $b_{i j}=0$ for all $i \leq j$, we may take $A=k$, and then (I1.2.3) is automatic. For every positive integer $d$, define

$$
S^{d}:=\left\{y_{1}^{d_{1}} \cdots y_{n}^{d_{n}} \cdots \mid \sum_{s} d_{s}=d\right\}
$$

The following lemma is known and easy to check by a direct computation.

Lemma 1.3. Suppose (I1.2.1)-(I1.2.3) hold.

(a) For every $n$,

$$
\Delta\left(y_{i}^{n}\right)=\sum_{s=0}^{n}\left(\begin{array}{l}
n \\
s
\end{array}\right)_{\lambda_{i i}} g_{i}^{s} y_{i}^{n-s} \otimes y_{i}^{s}+\sum_{s+s^{\prime}<n} a_{s s^{\prime}} y_{i}^{s} \otimes y_{i}^{s^{\prime}}
$$

for some $a_{s s^{\prime}} \in \sum_{t \geq 0} A g_{i}^{t}$. If $b_{i i}=0$, then $a_{s s^{\prime}}=0$ for all $s, s^{\prime}$. 
(b) Let $\left\{y_{1}, y_{2}, \cdots, y_{z}\right\}$ be a finite subset of $S$. Then, for $n_{1}, \cdots, n_{z} \geq 0$,

$$
\begin{aligned}
\Delta\left(y_{1}^{n_{1}} \cdots y_{z}^{n_{z}}\right)= & \sum_{s_{1}, \cdots, s_{z}}\left(\prod_{t=1}^{z}\left(\begin{array}{c}
n_{t} \\
s_{t}
\end{array}\right)_{\lambda_{t t}}\right) c_{\left(s_{t}\right)} g_{1}^{s_{1}} \cdots g_{z}^{s_{z}} y_{1}^{n_{1}-s_{1}} \cdots y_{z}^{n_{z}-s_{z}} \otimes y_{1}^{s_{1}} \cdots y_{z}^{s_{z}} \\
& +l d t_{2}
\end{aligned}
$$

where $c_{\left(s_{t}\right)}=\prod_{i<j} \lambda_{i j}^{s_{j}\left(n_{i}-s_{i}\right)} \in k^{\times}$. Here ldt $t_{2}$ is a linear combination of elements of the form $f y_{1}^{a_{1}} \cdots y_{z}^{a_{z}} \otimes y_{1}^{b_{1}} \cdots y_{z}^{b_{z}}$ with $\sum_{i}\left(a_{i}+b_{i}\right)<\sum_{i} n_{i}$, where $f \in \sum_{t_{1}, \cdots, t_{z} \geq 0} A g_{1}^{t_{1}} \cdots g_{z}^{t_{z}}$. If $b_{i j}=0$ for all $i \leq j$, then $l d t_{2}=0$.

For $\alpha=\left(n_{1}, \cdots, n_{z}, 0, \cdots\right)$, define

$$
L_{\alpha}=\sum_{G} D G
$$

where $G$ runs through elements $y_{1}^{m_{1}} \cdots y_{w}^{m_{w}}$ such that $\left(m_{1}, \cdots, m_{w}, 0, \cdots\right)<\alpha$.

Lemma 1.4. Retain the notation as above and suppose (I1.2.1)-(I1.2.3) hold. Let $\alpha=\left(n_{1}, \cdots, n_{z}, 0, \cdots\right)$ and $F=y_{1}^{n_{1}} \cdots y_{z}^{n_{z}}$. Define

$$
V=\left\{a \in D \mid a F \in L_{\alpha}\right\} .
$$

Then $V$ is either 0 or $D$.

Proof. Let $L$ denote $L_{\alpha}$ in the proof. First we claim that $\Delta(L) \subset L \otimes L$. It suffices to show that $\Delta(G) \in L \otimes L$ for all $G=y_{1}^{m_{1}} \cdots y_{w}^{m_{w}}$ with $\left(m_{1}, \cdots, m_{w}, 0, \cdots\right)<\alpha$. By Lemma 1.3.

$$
\Delta(G)=G \otimes 1+g_{1}^{m_{1}} \cdots g_{w}^{m_{w}} \otimes G+l d t_{2} \in L \otimes L .
$$

Thus we proved our claim. It is easy to see that the hypotheses in Lemma 1.1)(a, b) hold. For the hypothesis in Lemma 1.1(c), we note that

$$
\Delta(F)=F \otimes 1+g_{1}^{n_{1}} \cdots g_{z}^{n_{z}} \otimes F+l d t_{2}^{\prime}
$$

by Lemma 1.3 (b), where $l d t_{2}^{\prime} \in L \otimes L$. The assertion follows from Lemma 1.1 .

Here is the main result of this section. Recall that $g_{i}=\mu\left(y_{i}\right)$ for all $i$.

Theorem 1.5. Assume that (I1.2.1)-(I1.2.3) hold. Let $\lambda_{i}$ denote $\lambda_{i i}$ for all $i$.

(a) Suppose the elements in $\bigcup_{j \geq 0} S^{j}$ are linearly dependent over $D$ (on the left or on the right). Then there is some $z \in \mathbb{N}$ such that

(i) $\lambda_{z}$ is a primitive $p_{z}$-th root of unity for some $p_{z}>1$,

(ii) there are $a_{i}, b_{j} \in k, p_{j} \in \mathbb{N}$ such that $y_{z}^{p_{z}}+\sum_{i} a_{i} y_{i}+\sum_{j \neq z} b_{j} y_{j}^{p_{j}} \in D$,

(iii) $g_{i}=g_{z}^{p_{z}}$ whenever $a_{i} \neq 0$ in part (ii), and

(iv) $g_{j}^{p_{j}}=g_{z}^{p_{z}}$ and $\lambda_{j}$ is a primitive $p_{j}$-th root of unity whenever $b_{j} \neq 0$ in part (ii).

(b) Suppose $\lambda_{i}$ is either 1 or not a root of unity for every $i$. Then the elements in $\bigcup_{j \geq 0} S^{j}$ are linearly independent over $D$ (on the left and on the right). As a consequence,

$$
\operatorname{GKdim} H \geq \operatorname{GKdim} D+\#(S) .
$$

Proof. (a) Suppose that $\bigcup_{j \geq 0} S^{j}$ is linearly dependent over $D$ on the left. Then there is an $F=y_{1}^{n_{1}} \cdots y_{z}^{n_{z}} \in S^{d}$ for some $d \geq 0$ such that

$$
a F \in L_{\alpha}, \quad \text { for some } 0 \neq a \in D,
$$


where $\alpha=\left(n_{1}, \cdots, n_{z}, 0, \cdots\right)$. The definition of $L_{\alpha}$ is given in (11.3.1). Choose $F$ among all $(a, F)$ satisfying (I1.5.1) so that $\alpha$ is minimal with respect to the linear order $<$ defined at the beginning of Remark 1.2. For simplicity let $L=L_{\alpha}$ for the rest of the proof. Let $V=\{b \in D \mid b F \in L\}$. Then $0 \neq a \in V$. By Lemma 1.4. $1 \in V$ or, equivalently, $F \in L$. So we can write $F=l d t_{1}$ where $l d t_{1}$ denotes any element in $L$. By the minimality of $\alpha, L$ is a free left $D$-module with a basis $\left\{y_{1}^{m_{1}} \cdots y_{w}^{m_{w}} \mid\left(m_{1}, \cdots, m_{w}, 0, \cdots\right)<\alpha\right\}$. Note that $L \otimes L$ is a free $D \otimes D$-module with a basis

$$
\left\{y_{1}^{m_{1}} \cdots y_{w}^{m_{w}} \otimes y_{1}^{l_{1}} \cdots y_{w^{\prime}}^{l_{w^{\prime}}} \mid\left(m_{1}, \cdots, m_{w}, 0, \cdots\right),\left(l_{1}, \cdots, l_{w^{\prime}}, 0, \cdots\right)<\alpha\right\} .
$$

We define a multi-degree on $L$ such that, for any nonzero $a \in D, \operatorname{deg}(a)=0$ and $\operatorname{deg}\left(a y_{1}^{m_{1}} \cdots y_{w}^{m_{w}}\right)=\left(m_{1}, \cdots, m_{w}, 0, \cdots\right)$ whenever $\left(m_{1}, \cdots, m_{w}, 0, \cdots\right)<\alpha$. Notice that under this definition $L$ is a graded $D$-module (but not an algebra) which can obviously be viewed as a filtered $D$-module. Extend this multi-grading naturally to $L \otimes L$ by adding the multi-degrees of the tensor components.

Recall that $F=y_{1}^{n_{1}} \cdots y_{z}^{n_{z}}$. We may assume $n_{z}>0$ (if not, delete $y_{z}$ in the expression of $F$ ). Following the last paragraph, there is an $l d t_{1} \in L$ such that $F=-l d t_{1}$, or equivalently, $y_{1}^{n_{1}} \cdots y_{z}^{n_{z}}+l d t_{1}=0$. By the choice of $F$, any element in $L$ has multi-degree less than $\alpha$. Let $l d t_{2}$ denote any element in $L \otimes L$ and let $l m t_{2}$ denote any element in $L \otimes L$ with multi-degree less than $\alpha$. Since the multi-degree of $l d t_{1}$ is less than $\alpha, \Delta\left(l d t_{1}\right)$ is an $l m t_{2}$ by Lemma 1.3. Then, by Lemma 1.3 again, we have

$$
\begin{aligned}
0= & \Delta\left(F+l d t_{1}\right)=\Delta(F)+l m t_{2} \\
= & \sum_{s_{1}, \cdots, s_{z}}\left(\prod_{t=1}^{z}\left(\begin{array}{c}
n_{t} \\
s_{t}
\end{array}\right)_{\lambda_{t}}\right) c_{\left(s_{t}\right)} g_{1}^{s_{1}} \cdots g_{z}^{s_{z}} y_{1}^{n_{1}-s_{1}} \cdots y_{z}^{n_{z}-s_{z}} \otimes y_{1}^{s_{1}} \cdots y_{z}^{s_{z}}+l m t_{2} \\
= & \sum_{\left(s_{t}\right) \neq(0),\left(n_{t}\right)}\left(\prod_{t=1}^{z}\left(\begin{array}{c}
n_{t} \\
s_{t}
\end{array}\right)_{\lambda_{t}}\right) c_{\left(s_{t}\right)} g_{1}^{s_{1}} \cdots g_{z}^{s_{z}} y_{1}^{n_{1}-s_{1}} \cdots y_{z}^{n_{z}-s_{z}} \otimes y_{1}^{s_{1}} \cdots y_{z}^{s_{z}} \\
& \quad+F \otimes 1+g_{1}^{n_{1}} \cdots g_{z}^{n_{z}} \otimes F+l m t_{2} \\
& \quad \sum_{\left(s_{t}\right) \neq(0),\left(n_{t}\right)}\left(\prod_{t=1}^{z}\left(\begin{array}{c}
n_{t} \\
s_{t}
\end{array}\right)_{\lambda_{t}}\right) c_{\left(s_{t}\right)} g_{1}^{s_{1}} \cdots g_{z}^{s_{z}} y_{1}^{n_{1}-s_{1}} \cdots y_{z}^{n_{z}-s_{z}} \otimes y_{1}^{s_{1}} \cdots y_{z}^{s_{z}}+l m t_{2},
\end{aligned}
$$

where $l m t_{2}$ represents an element in $L \otimes L$ with multi-degree less than $\alpha$. The multi-degree of $g_{1}^{n_{1}} \cdots g_{z}^{n_{z}} y_{1}^{n_{1}-s_{1}} \cdots y_{z}^{n_{z}-s_{z}} \otimes y_{1}^{s_{1}} \cdots y_{z}^{s_{z}}$ equals $\alpha$ for any $\left(s_{t}\right) \neq$ $(0),\left(n_{t}\right)$. Using the fact that $L$ is a free $D$-module with basis $\left\{y_{1}^{m_{1}} \cdots y_{w}^{m_{w}}\right.$ । $\left.\left(m_{1}, \cdots, m_{w}, 0, \cdots\right)<\alpha\right\}$, we obtain that $\left(\prod_{t=1}^{z}\left(\begin{array}{l}n_{t} \\ s_{t}\end{array}\right)_{\lambda_{t}}\right) c_{\left(s_{t}\right)}=0$ or $\prod_{t=1}^{z}\left(\begin{array}{l}n_{t} \\ s_{t}\end{array}\right)_{\lambda_{t}}=0$ for all $\left(s_{t}\right) \neq(0),\left(n_{t}\right)$. If $n_{j}>0$ for some $1 \leq j<z$, we take $\left(s_{t}\right)=\left(0,0, \cdots, 0, n_{z}\right)$, then $\prod_{t=1}^{z}\left(\begin{array}{l}n_{t} \\ s_{t}\end{array}\right)_{\lambda_{t}}=1$, a contradiction. Therefore $n_{j}=0$ for all $j<z$, which means that $F=y_{z}^{n_{z}}$.

If $n_{z}=1$, we have $y_{z}=\sum_{i<z} b_{i} y_{i}+c$ for $c, b_{i} \in D$. Hence $\sum_{i<z} b_{i} y_{i}+c$ is $\left(1, g_{z}\right)$-primitive. Then applying $\Delta$ we obtain that

$$
\begin{aligned}
\Delta\left(b_{i}\right) & =b_{i} \otimes 1, \\
\Delta\left(b_{i}\right)\left(g_{i} \otimes 1\right) & =g_{z} \otimes b_{i}, \\
\Delta(c) & =c \otimes 1+g_{z} \otimes c .
\end{aligned}
$$


These imply that $b_{i} \in k$ and $g_{i}=g_{z}$ when $b_{i} \neq 0$. This contradicts (I1.2.2). Therefore $n_{z}>1$.

By the last two paragraphs, $n_{z}>1$ and $n_{i}=0$ for all $i<z$ and $\left(\begin{array}{l}n_{z} \\ s_{z}\end{array}\right)_{\lambda_{z}}=0$ for all $1 \leq s_{z} \leq n_{z}-1$. This can only happen when $\lambda_{z}$ is a primitive $n_{z}$-th root of unity [GZ2, Lemma 7.5].

Next let us re-name $n_{z}$ by $p_{z}$ and write $F=y_{z}^{p_{z}}$. Then $y_{z}^{p_{z}}+\sum_{i} b_{i} G_{i}+c_{0}=$ 0 , where $b_{i}, c_{0} \in D$ and the $G_{i}$ are monomials with multi-y-degree less than $\left(0, \cdots, 0, p_{z}, 0, \cdots\right)$ (where $p_{z}$ is in the $z$-th position). Repeating a computation similar to (11.5.2) (and the induction on the multi- $y$-degree of $G_{i}$ ) one can show that each $G_{i}$ (when $b_{i} \neq 0$ ) is of the form $y_{i}^{n_{i}}$ and each $y_{i}^{n_{i}}$ is a skew primitive. If $n_{i}>1$, then $\lambda_{i}$ is a primitive $n_{i}$-th root of unity. In summary, when $\lambda_{i}$ is not a root of unity, then $n_{i}=1$, and when $\lambda_{i}$ is a primitive $p_{i}$-th root of unity, then $n_{i}$ is either 1 or $p_{i}$. So we have

$$
-y_{z}^{p_{z}}=\sum_{i} a_{i} y_{i}+\sum_{j \neq z} b_{j} y_{j}^{p_{j}}+c,
$$

where $0 \neq a_{i}, b_{j} \in D$ and $c \in D$. Thus $\sum_{i} a_{i} y_{i}+\sum_{j} b_{j} y_{j}^{p_{j}}+c$ is $\left(1, g_{z}^{p_{z}}\right)$-primitive. Since $L$ is a free left $D$-module, each of the nonzero $a_{i} y_{i}, b_{j} y_{j}^{p_{j}}$ and $c$ is $\left(1, g_{z}^{p_{z}}\right)$ primitive. The coproduct computation shows that $a_{i}, b_{j} \in k$ and $g_{i}=g_{z}^{p_{z}}$ and $g_{j}^{p_{j}}=g_{z}^{p_{z}}$.

(b) The first assertion is an immediate consequence of part (a). To prove the second assertion, we take $W$ to be a finite dimensional subspace of $D$ and let $S$ be a finite set $\left\{y_{1}, \cdots, y_{z}\right\}$. For a subspace $V \subset H$, let $V^{n}$ be the linear span of all elements $v_{1} \cdots v_{n}$ for $v_{i} \in V$. By the first assertion,

$$
\begin{aligned}
\operatorname{dim}\left(W+k 1+\sum_{i=1}^{z} k y_{i}\right)^{2 n} & \geq \operatorname{dim} W^{n}\left(k 1+\sum_{i=1}^{z} k y_{i}\right)^{n} \\
& \geq\left(\operatorname{dim} W^{n}\right) \#\left(\bigcup_{d=0}^{n} S^{d}\right) \geq\left(\operatorname{dim} W^{n}\right) c n^{z}
\end{aligned}
$$

for some positive constant $c$. This implies that $\operatorname{GKdim} H \geq \operatorname{GKdim} D+\# S$. If $S$ is infinite, let $S^{\prime}$ be any finite subset of $S$. Then the above argument shows that $\operatorname{GKdim} H \geq \operatorname{GKdim} D+\# S^{\prime}$ for any $S^{\prime}$. Thus $\operatorname{GKdim} H=\infty=\operatorname{GKdim} D+$ $\# S$.

Proof of Theorem 0.2. Let $S=\left\{y_{1}, \cdots, y_{w}\right\}$. Then (I1.2.1)-(I1.2.3) follow easily from (a) and (b). The hypothesis in Theorem 1.5(b) is the same as that in Theorem [0.2(c). Therefore the assertion follows from Theorem 1.5 (b).

The following easy lemma will be used implicitly later.

Lemma 1.6. Let $P$ be the set of all skew primitive elements in a Hopf algebra $H$ with weight $\mu$. Then $P$ is a $k$-subspace of $H$ and $P \cap C_{0}=k(\mu-1)$.

Proof. It is clear that $P$ is a $k$-subspace of $H$. For any element $y \in P \cap C_{0}$, write $y=\sum_{i=1}^{n} c_{i} g_{i}$ for some $c_{i} \in k$ and $g_{i} \in G(H)$. Then the equation $\Delta(y)=y \otimes 1+\mu \otimes y$ forces the fact that $y \in k(\mu-1)$. 


\section{SECOND LOWER BOUnd THEOREM}

In this section we prove Theorem 0.3 , which is a consequence of Theorem $0.2, \mathrm{~A}$ stronger version will be proved in the next section. Lemmas presented here are also needed for the next section and cannot be omitted even if we skip Theorem 0.3 . If GKdim $H=\infty$, then Theorem 0.3 is vacuous. So we may assume that GKdim $H<$ $\infty$. We refer to Section 0 for the definitions of $W, \Omega, \Gamma$ and $W_{\sqrt{ }}, \Omega_{\sqrt{ }}, \Gamma_{\sqrt{ }}$.

Lemma 2.1. Let $y$ be a skew primitive element not in $C_{0}$ such that $\gamma(y)$ is defined.

(a) If $\gamma(y) \in \Gamma \backslash \Gamma_{\sqrt{ }}$, then $y^{n}$ is not skew primitive for any $n>1$.

(b) If $\gamma(y) \in \Gamma_{\sqrt{ }}$, then $\mu(y) \in W_{\sqrt{ }}$.

Proof. (a) Take $S$ to be the singleton $\{y\}$ and $D=C_{0}$. Then (I1.2.1)-(I1.2.3) hold for $A=k\left[\mu(y)^{ \pm 1}\right]$. Since $\gamma(y) \in \Gamma \backslash \Gamma_{\sqrt{ }}$ the hypothesis in Theorem [1.5 (b) holds. By Theorem 1.5(b), $\left\{y^{n}\right\}_{n \geq 0}$ is linearly independent over $C_{0}$. Since $\gamma(y)$ is not a root of unity, for any $n>1, \Delta\left(y^{n}\right) \notin H \otimes k+C_{0} \otimes H$ by Lemma 1.3(a). Thus $y^{n}$ is not a skew primitive. The assertion follows.

(b) Suppose $\gamma(y) \in \Gamma_{\sqrt{ }}$. Since $\gamma(y) \neq 1$, replacing $y$ by $y+\alpha(\mu(y)-1)$ for a suitable $\alpha \in k$, we have $\mu(y)^{-1} y \mu(y)=\gamma(y) y$. Since $\gamma(y)$ is a primitive $n$-th root of unity for some $n>1$, Lemma 1.3(a) says that $\Delta\left(y^{n}\right)=y^{n} \otimes 1+\mu(y)^{n} \otimes y^{n}$, which means that $y^{n}$ is a skew primitive (could be zero). Therefore $\mu(y) \in W_{\sqrt{ }}$.

Let $G(H)$ denote the group of all group-like elements in a Hopf algebra $H$. Recall that $\operatorname{GKdim} H<\infty$ by a general assumption in this section.

Lemma 2.2. Let $y$ be a skew primitive element not in $C_{0}$ and let $x=\mu(y)$. Suppose $\gamma(y)$ exists. Assume that $G_{0}$ is a subgroup of $G(H)$ commuting with $x$. Let $V=k(x-1)+\sum_{g \in G_{0}} k\left(g^{-1} y g\right)$.

(a) Every $z \in V$ is $(1, x)$-primitive, and $\omega(z)=\omega(y)$ for all $z \in V \backslash k(x-1)$.

(b) If $\gamma(y) \in \Gamma \backslash \Gamma_{\sqrt{ }}$, then $\operatorname{dim} V \leq \mathrm{GKdim} H-\mathrm{GKdim} C_{0}+1$.

(c) Suppose that $V$ is finite dimensional and that $G_{0}$ is abelian. Then there is $z \in V \backslash k(x-1)$ such that either

(ci) for every $g \in G_{0}, g^{-1} z g=\lambda_{g} z$ for some $\lambda_{g} \in k^{\times}$or

(cii) for every $g \in G_{0}, g^{-1} z g=z+\tau_{g}(x-1)$ for some $\tau_{g} \in k$.

(d) If $\gamma(y)$ is not a root of unity, then $\mu(y)$ has infinite order.

Proof. (a) Since $g x=x g$ for all $g \in G_{0}, g^{-1} y g$ is a $(1, x)$-primitive with $\omega\left(g^{-1} y g\right)=$ $\omega(y)$.

(b) Let $S=\left\{g_{i}^{-1} y g_{i}\right\}_{i=1}^{w}$ be a finite subset of $V$ which is linearly independent in the space $V / k(x-1)$. Here $g_{i} \in G_{0}$ for all $i=1, \cdots, w$. For different $i$, we have $\mu\left(g_{i}^{-1} y g_{i}\right)=x$, and

$$
x^{-1}\left(g_{i}^{-1} y g_{i}\right) x=g_{i}^{-1}\left(x^{-1} y x\right) g_{i}=\gamma(y)\left(g_{i}^{-1} y g_{i}\right)+\tau(x-1)
$$

where $\tau$ is the same as the one in (I0.3.3). Then the hypotheses (I1.2.1)-(I1.2.3) hold for $A=k\left[x^{ \pm 1}\right]$ and $D=C_{0}$. Since $\lambda:=\gamma(y)$ is either 1 or not a root of unity, Theorem 1.5(b) says that $\# S \leq \operatorname{GKdim} H-\operatorname{GKdim} C_{0}$. Clearly $V \cap C_{0}=k(x-1)$. Thus

$$
\operatorname{dim} V-1=\operatorname{dim} V /\left(V \cap C_{0}\right)=\# S \leq \operatorname{GKdim} H-\operatorname{GKdim} C_{0},
$$

since $S$ is a basis of $V /\left(V \cap C_{0}\right)$.

(c) First we may assume $x \in G_{0}$. If not, replace $G_{0}$ by the subgroup generated by $G_{0}$ and $x$ (replacing $G_{0}$ by this larger subgroup does not enlarge $V$, because of 
(10.3.3) $)$. Then $V$ is a $G_{0}$-module by conjugation action. Since $G_{0}$ is abelian and $k$ is algebraically closed, every finite dimensional simple $G_{0}$-module is 1-dimensional. Thus $V$ has a 1-dimensional simple $G_{0}$-submodule $k z$. If $z \notin k(x-1)$, then $k z$ being a simple $G_{0}$-module is equivalent to (ci). Otherwise, no element $z \in V \backslash k(x-1)$ generates a simple $G_{0}$-submodule. Hence $V$ has a unique simple $G_{0}$-submodule $M_{0}:=k(x-1)$. Note that $g^{-1}(x-1) g=(x-1)$ for all $g \in G_{0}$, so $M_{0}$ is the trivial $G_{0}$-module. Since $G_{0}$ is commutative and $V$ has only one simple submodule, every simple subquotient of $V$ must be isomorphic to the simple $M_{0}$. Pick $z \in V \backslash k(x-1)$ so that the submodule $M$ generated by $z$ is 2 -dimensional. Then $M / k(x-1) \cong M_{0}$, which says that $g^{-1} z g \equiv z$ modulo $k(x-1)$. Hence $g^{-1} z g=z+\tau_{g}(x-1)$ for some $\tau_{g} \in k$.

(d) Let $G_{0}=\langle\mu(y)\rangle$. It follows from the definition that the existence of $\gamma(y)$ implies that $V$ is finite dimensional. Applying part (ci) to the cyclic group $G_{0}$ there is a skew primitive $z \in H \backslash C_{0}$ such that

$$
g^{-1} z g=\lambda(g) z
$$

for all $g \in G_{0}$. It is also clear that $\lambda(\mu(y))=\gamma(y)$. Since $\gamma(y)$ is not a root of unity, the image of $\lambda: G_{0} \rightarrow k^{\times}$is infinite. Consequently, $G_{0}$ is infinite and $\mu(y)$ has infinite order.

Lemma 2.3. Let $\left\{z_{i}\right\}_{i=1}^{w}$ be a set of skew primitive elements not in $C_{0}$ such that $\gamma\left(z_{i}\right)$ exists for each $i$. If the elements $\omega\left(z_{1}\right), \cdots, \omega\left(z_{w}\right)$ are distinct, then $\left\{z_{i}\right\}_{i=1}^{w}$ is linearly independent in $H / C_{0}$.

Proof. Suppose $\left\{z_{i}\right\}_{i=1}^{w}$ is linearly dependent in $H / C_{0}$. Pick a minimal subset, say $\left\{z_{j}\right\}_{j=1}^{v}$, such that $\sum_{j=1}^{\bar{v}} a_{j} z_{j}=: c \in C_{0}$ for some scalars $a_{j} \in k^{\times}$. Thus $v>1$ since $z_{i} \notin C_{0}$ for any $i$. Applying $\Delta$ to the equation $\sum_{j=1}^{v} a_{j} z_{j}=c$, we have

$$
\Delta(c)=\Delta\left(\sum_{j=1}^{v} a_{j} z_{j}\right)=\sum_{j=1}^{v} a_{j} z_{j} \otimes 1+\sum_{j=1}^{v} a_{j} \mu\left(z_{j}\right) \otimes z_{j}=c \otimes 1+\sum_{j=1}^{v} a_{j} \mu\left(z_{j}\right) \otimes z_{j} .
$$

Hence $\sum_{j=1}^{v} \mu\left(z_{j}\right) \otimes a_{j} z_{j} \in C_{0} \otimes C_{0}$. By the minimality of $v, \mu\left(z_{j}\right)=\mu\left(z_{j^{\prime}}\right)$ for all $j, j^{\prime}$.

Set $x=\mu\left(z_{j}\right)$ for all $1 \leq j \leq v$. Applying the conjugation by $x$ to the equation $\sum_{j=1}^{v} a_{j} z_{j}=c$, we obtain $\sum_{j=1}^{v} \gamma\left(z_{j}\right) a_{j} z_{j}=-\sum_{j=1}^{v} \tau_{j}(x-1)+x^{-1} c x \in C_{0}$ for some $\tau_{j} \in k$. Using the minimality of $v, \gamma\left(z_{j}\right)=\gamma\left(z_{j^{\prime}}\right)$ for all $j, j^{\prime}$. Thus we obtain a contradiction. The assertion follows.

Theorem 2.4. Let $\left\{y_{i}\right\}_{i=1}^{w}$ be a set of skew primitive elements not in $C_{0}$ such that $\omega\left(y_{1}\right), \cdots, \omega\left(y_{w}\right)$ are defined and distinct elements in $\Omega \backslash \Omega_{\sqrt{ }}$. If the subgroup $G_{0}$ generated by $\left\{\mu\left(y_{i}\right)\right\}_{i=1}^{w}$ is abelian, then $\operatorname{GKdim} H \geq \operatorname{GKdim} C_{0}+w$.

Proof. By LemmaL2.2(a, c), for each $i$ there is a $z_{i}$ in $k\left(\mu\left(y_{i}\right)-1\right)+\sum_{g \in G_{0}} k g^{-1} y_{i} g$ but not in $C_{0}$ such that

$$
\omega\left(z_{i}\right)=\omega\left(y_{i}\right)
$$

and, for every $g \in G_{0}$,

$$
g^{-1} z_{i} g=\lambda_{i g} z_{i}+\tau_{i g}\left(\mu\left(y_{i}\right)-1\right)
$$

for some $\lambda_{i g} \in k^{\times}, \tau_{i g} \in k$. Let $A=k G_{0}$ and $D=C_{0}$. Then (I1.2.1) is clear and (I1.2.2) follows from Lemma 2.3 for the set $\left\{z_{i}\right\}_{i=1}^{w}$. (I1.2.3) is a consequence of Lemma 2.2(c), as we have already seen. By hypothesis, each $\lambda_{i}:=\gamma\left(z_{i}\right)$ is either 1 
or not a root of unity. Therefore GKdim $H \geq \operatorname{GKdim} C_{0}+w$ by applying Theorem 1.5(b) to the set $\left\{z_{i}\right\}_{i=1}^{w}$.

The next lemma is a result of [Zhu]. As before, we assume that GKdim $H<\infty$, which is one of the hypotheses in [Zhu, Theorem 1.2].

Lemma 2.5 ( $\left[\mathrm{Zhu}\right.$, Theorem 1.2]). Let $y$ be a skew primitive element not in $C_{0}$ with $g=\mu(y)$. Then there is a skew primitive element $z=\sum_{i=0}^{n} b_{i} g^{-i} y g^{i} \in H \backslash C_{0}$, where $b_{i} \in k$, such that $g^{-1} z g=\lambda z+\tau(g-1)$ for some $\lambda \in k^{\times}$and $\tau \in k$. Further, if $\lambda \neq 1$, then there is $z^{\prime}=z+\alpha(g-1)$ for a suitable $\alpha \in k$ such that $g^{-1} z^{\prime} g=\lambda z^{\prime}$.

Proof. In [Zhu, Theorem 1.2] $H$ is assumed to be pointed, but the statement is valid without this hypothesis. The first assertion is equivalent to $\mathrm{Zhu}$, Theorem 1.2]. If $\lambda \neq 1$, take $\alpha=(\lambda-1)^{-1} \tau$. Then $z^{\prime}=z+\alpha(g-1)$ is a $(1, g)$-primitive element satisfying $g^{-1} z^{\prime} g=\lambda z^{\prime}$.

Now we are ready to prove Theorem 0.3 .

Proof of Theorem 0.3. Pick any finite subset $\left\{\mu\left(y_{i}\right)\right\}_{i=1}^{w}$ of $W \backslash W_{\sqrt{ }}$ where each $y_{i}$ is a skew primitive not in $C_{0}$. By Lemma 2.5. for each $i$ there is a skew primitive $y_{i}^{\prime}$ not in $C_{0}$ such that $g_{i}:=\mu\left(y_{i}^{\prime}\right)=\mu\left(y_{i}\right)$ and that $\gamma\left(y_{i}^{\prime}\right)$ is defined. By Lemma 2.1(b), $\gamma\left(y_{i}^{\prime}\right)$ is not a root of unity or 1 . Hence $\omega\left(y_{i}^{\prime}\right) \in \Omega \backslash \Omega_{\sqrt{ }}$. The assertion follows from Theorem 2.4

Theorem 2.4 shows in fact that if $\left\langle W \backslash W_{\sqrt{ }}\right\rangle$ is abelian, then

$$
\operatorname{GKdim} H \geq \operatorname{GKdim} C_{0}+\#\left(\Omega \backslash \Omega_{\sqrt{ }}\right) .
$$

There is also an inequality

$$
\operatorname{GK} \operatorname{dim} H \geq \operatorname{GKdim} C_{0}+\#\left(W^{\prime}\right)
$$

for any $W^{\prime} \subset W \backslash W_{\sqrt{ }}$ such that $\left\langle W^{\prime}\right\rangle$ is abelian.

Suppose there is a surjective Hopf algebra morphism $\pi: H \rightarrow C_{0}$ such that the restriction to $C_{0}$ is the identity. Let $A$ be the subalgebra of $H$ generated by all skew primitive elements in $\operatorname{ker} \pi$, and let $G_{W}$ be the subsemigroup of $G(H)$ generated by $\mu(y)$ for all skew primitive elements $y \in A$. We do not assume that $G_{W}$ is abelian.

Lemma 2.6. Suppose there is a surjective Hopf algebra morphism $\pi: H \rightarrow C_{0}$ such that the restriction to $C_{0}$ is the identity. Let $A$ be defined as above.

(a) $H=R \# C_{0}$, where $R$ is the ring of right coinvariants of $\pi$. Then $A$ is a subalgebra of $R$ and

$\operatorname{GKdim} H \geq \operatorname{GKdim} R+\operatorname{GKdim} C_{0} \geq \operatorname{GKdim} A+\operatorname{GKdim} C_{0}$.

(b) Assume that $A$ is a domain. Then $\operatorname{GKdim} A \geq \operatorname{GKdim} k G_{W}$. As a consequence,

$$
\operatorname{GKdim} H \geq \operatorname{GKdim} k G_{W}+\operatorname{GKdim} C_{0} .
$$

Proof. (a) By [Mo, Theorem 7.2.2], $H$ is isomorphic to a crossed product $R \#_{\sigma} C_{0}$ as algebras, and by $\mathrm{Mo}$, Proposition 7.2.3], $\sigma$ is trivial. Hence $H=R \# C_{0}$, where $R$ is the ring of right coinvariants of $\pi$. It is clear that every skew primitive element in $\operatorname{ker} \pi$ is in $R$. Therefore $A \subset R$.

Since $H=R \# C_{0}, G K \operatorname{dim} H \geq \operatorname{GKdim} R+\operatorname{GKdim} C_{0}$. The assertion follows by the fact $A \subset R$. 
(b) Define a map $\rho: A \rightarrow C_{0} \otimes H$ to be the composition $\left(\pi \otimes I d_{H}\right) \circ \Delta$. Since $\rho(y) \in k G_{W} \otimes A$ for all skew primitive elements $y \in A$ and since $A$ is generated by these $y$ 's, the image of $\rho$ is in $k G_{W} \otimes A$. Consequently, $(A, \rho)$ is a left $k G_{W}$-comodule algebra. This means that $A$ is a $G_{W}$-graded algebra. Let $f: A \rightarrow C_{0}$ be the map sending any nonzero homogeneous element $h \in A$ to its degree, for example, sending $y_{1} \cdots y_{n}$ to $\mu\left(y_{1}\right) \cdots \mu\left(y_{n}\right)$. Since $A$ is a domain, $f$ is multiplicative.

Pick any finite dimensional space $V=k+\sum_{i=1}^{m} k \mu\left(y_{i}\right)$ of $k G_{W}$ where the $y_{i}$ are skew primitive elements in $A$, and let $W=\{1\} \cup\left\{y_{i}\right\}_{i=1}^{w}$. Then $\operatorname{dim}(k W)^{n} \geq$ $\#\left(f\left(W^{n}\right)\right) \geq \#(f(W))^{n} \geq \operatorname{dim} V^{n}$ for all $n$. Hence $\operatorname{GKdim} A \geq \operatorname{GKdim} k G_{W}$.

The next example shows why we need to remove $W_{\sqrt{ }}$ from $W$ (or remove $\Gamma_{\sqrt{ }}$ from $\Gamma$ ) in the lower bound theorems.

Example 2.7. Let $B$ be the Hopf algebra $B\left(1,1, p_{1}, \cdots, p_{s}, q\right)$ defined in GZ2, Construction 1.2]. This is a finitely generated, noetherian, pointed Hopf domain of GK-dimension 2. By [GZ2, Construction 1.2] $B$ is generated by $x, x^{-1}, y_{1}, \cdots, y_{s}$, where $x$ is a group-like element and the $y_{i}$ 's are skew primitive elements. Let $z=y_{1}^{p_{1}}$. Then $z=y_{j}^{p_{j}}$ for all $j$ and it is a central skew primitive element. Let $H=B /\left(z, x^{m}-1\right)$, where $m=\prod_{i} p_{i}$. Then $H$ is a finite dimensional pointed Hopf algebra of GK-dimension 0 and $C_{0}=k\left[x, x^{-1}\right] /\left(x^{m}-1\right)$ has GK-dimension 0.

By [GZ2, Construction 1.2], $W=W_{\sqrt{ }}=\left\{x^{m_{i}}\right\}_{i=1}^{s}$, where $m_{i}=m / p_{i}, \Gamma=$ $\Gamma_{\sqrt{ }}=\left\{q^{-m_{i}^{2}}\right\}_{i=1}^{s}, \Omega=\Omega_{\sqrt{ }}=\left\{\left(x^{m_{i}}, q^{-m_{i}^{2}}\right)\right\}_{i=1}^{s}$, and $Z=Y_{\sqrt{ }}+C_{0}$ and $Y_{\sqrt{ }}=$ $\sum_{i=1}^{s} k y_{i}$. Thus

$$
\#\left(W_{\sqrt{ }}\right)=\#\left(\Gamma_{\sqrt{ }}\right)=\#\left(\Omega_{\sqrt{ }}\right)=\operatorname{dim} Y_{\sqrt{ }}=s
$$

which can be arbitrarily large.

\section{THIRD LOWER BOUND THEOREM}

The first half of this section concerns some preliminary analysis of Hopf algebras with exponential growth and the proof of Proposition 0.5. The proof of the third lower bound theorem is given at the end of the section.

Let $\left(G_{0}, \times\right)$ be a multiplicative abelian group and $\Lambda:=\left\{\lambda_{1}, \cdots, \lambda_{v}\right\}$ be a list of 1-dimensional group representations of $G_{0}$ for some $v>1$. Note that this list is allowed to have repetitions. When some $\lambda_{i}$ is the trivial representation of $G_{0}$ (namely, $\lambda_{i}(g)=1$ for all $g \in G_{0}$ ), then we also need a group homomorphism $\tau_{i}:\left(G_{0}, \times\right) \rightarrow(k,+)$ (which must be zero if $G_{0}$ is torsion, since char $k=0$ ). When $\lambda_{i}$ is not trivial, we set $\tau_{i}=0$.

Now pick a list of elements $\mu:=\left\{\mu_{1}, \cdots, \mu_{v}\right\}$ in $G_{0}$ (again allowing repetitions). Let $K:=K(\Lambda, \mu)$ be the Hopf algebra generated as an algebra by the elements in the abelian group $G_{0}$ and a set of skew primitive elements $y_{1}, \cdots, y_{v}$ subject to the relations within $G_{0}$ and the following additional relations between $G_{0}$ and $\left\{y_{i}\right\}_{s=1}^{v}$ :

$$
y_{i} g=\lambda_{i}(g) g y_{i}+\tau_{i}(g) g\left(\mu_{i}-1\right) \text {, for all } i \text { and all } g \in G_{0} .
$$

The coalgebra structure of $K$ is determined by

$$
\begin{aligned}
\Delta(g)=g \otimes g, & \epsilon(g)=1, \quad \text { for all } g \in G_{0}, \\
\Delta\left(y_{i}\right)=y_{i} \otimes 1+\mu_{i} \otimes y_{i}, & \epsilon\left(y_{i}\right)=0, \quad \text { for all } i=1, \cdots, v .
\end{aligned}
$$


And the antipode of $K$ is determined by

$$
\begin{aligned}
S(g) & =g^{-1}, \quad \text { for all } g \in G_{0}, \\
S\left(y_{i}\right) & =-\mu_{i}^{-1} y_{i}, \quad \text { for all } i=1, \cdots, v .
\end{aligned}
$$

Let $\lambda_{i j}=\lambda_{i}\left(\mu_{j}\right)$ for all $i, j$, and let $\Lambda_{M}$ be the $v \times v$-matrix $\left(\lambda_{i j}\right)$.

By Remark 1.2, the total $y$-degree and the multi- $y$-degree are defined for elements in $K$. For example, the (filtered) multi- $y$-degree of $g y_{3} y_{2}$ is $(0,1,1,0, \cdots, 0) \in \mathbb{N}^{v}$.

Let $F$ be a nonzero skew primitive element in $K$ with total $y$-degree $z \geq 2$. Write $F=\sum c_{h,\left(i_{s}\right)} h y_{i_{1}} y_{i_{2}} \cdots y_{i_{n}}$, where $h \in G_{0}$ and $0 \neq c_{h,\left(i_{s}\right)} \in k$. A term of $F$ means a nonzero monomial $c_{h,\left(i_{s}\right)} h y_{i_{1}} y_{i_{2}} \cdots y_{i_{n}}$ appearing in $F$.

Lemma 3.1. Let $K:=K(\Lambda, \mu)$ be defined as above.

(a) $K$ has a $k$-linear basis

$$
\left\{g y_{i_{1}} y_{i_{2}} \cdots y_{i_{s}}\right\}
$$

where $g \in G_{0}, i_{1}, \cdots, i_{s} \in\{1, \cdots, v\}$. As a consequence, $K$ contains a free subalgebra $k\left\langle y_{1}, y_{2}\right\rangle$ and has exponential growth.

(b) The coradical of $K$ is $k G_{0}$.

(c) If $F$ is a skew primitive element of total $y$-degree $z \geq 2$, then for any term of $F$ with multi-y-degree $\left(N_{1}, \cdots, N_{v}\right)$ and $\sum_{i} N_{i}=z$,

$$
\prod_{i=1}^{v}\left(\lambda_{i i}\right)^{N_{i}\left(N_{i}-1\right)} \prod_{i<j}\left(\lambda_{i j} \lambda_{j i}\right)^{N_{i} N_{j}}=1 .
$$

Proof. (a) The first assertion follows from Bergman's Diamond Lemma Be, Theorem 1.2]. Consequently, $K$ contains the free algebra of rank $2, k\left\langle y_{1}, y_{2}\right\rangle$. Therefore $K$ has exponential growth.

(b) By definition, $\Delta$ is compatible with filtrations defined in Remark 1.2. Hence $\Delta$ is a homomorphism of filtered algebras. So every group-like element must have total $y$-degree 0 . The assertion follows.

(c) Let $F=\sum c_{h,\left(i_{s}\right)} h y_{i_{1}} y_{i_{2}} \cdots y_{i_{n}}$ with coefficients $c_{h,\left(i_{s}\right)} \neq 0$. For simplicity, let $l d t$ denote any linear combination of monomials of total $y$-degree less than $z$. Then we can write $F=\sum c_{h,\left(i_{s}\right)} h y_{i_{1}} y_{i_{2}} \cdots y_{i_{z}}+l d t$. Since $\Delta(F)=F \otimes 1+\mu(F) \otimes$ $F, h=1$ for terms with total degree $z$. Pick any term of $y$-degree $z$ in $F$, say $c_{1,\left(i_{s}\right)} y_{i_{1}} y_{i_{2}} \cdots y_{i_{z}}$, and let $\left(N_{1}, \cdots, N_{v}\right)$ be its multi- $y$-degree.

Since $F$ is skew primitive, $S(F)=-\mu(F)^{-1} F$. Since $S\left(y_{i}\right)=-\mu_{i}^{-1} y_{i}$, we have

$$
\begin{aligned}
S\left(c_{1,\left(i_{s}\right)} y_{i_{1}} \cdots y_{i_{z}}\right) & =c_{1,\left(i_{s}\right)}\left(-\mu_{i_{z}}^{-1} y_{i_{z}}\right) \cdots\left(-\mu_{i_{1}}^{-1} y_{i_{1}}\right) \\
& =c_{1,\left(i_{s}\right)}(-1)^{z} \prod_{s>t} \lambda_{i_{s} i_{t}}^{-1} \mu^{-1} y_{i_{z}} \cdots y_{i_{1}}+l d t
\end{aligned}
$$

where $\mu=\prod_{s=1}^{z} \mu_{i_{s}}$. Since $S(F)=-\mu(F)^{-1} F, \mu=\mu(F)$ and $F$ contains a nonzero term of the form $c_{\left(i_{s}\right)}^{\prime} y_{i_{z}} \cdots y_{i_{1}}$. The same computation shows that

$$
\begin{aligned}
S\left(c_{\left(i_{s}\right)}^{\prime} y_{i_{z}} \cdots y_{i_{1}}\right) & =c_{\left(i_{s}\right)}^{\prime}\left(-\mu_{i_{1}}^{-1} y_{i_{1}}\right) \cdots\left(-\mu_{i_{z}}^{-1} y_{i_{z}}\right) \\
& =c_{\left(i_{s}\right)}^{\prime}(-1)^{z} \prod_{a<b} \lambda_{i_{a} i_{b}}^{-1} \mu^{-1} y_{i_{1}} \cdots y_{i_{z}}+l d t .
\end{aligned}
$$


Comparing the coefficients in the terms $\mu^{-1} y_{i_{z}} \cdots y_{i_{1}}$ and $\mu^{-1} y_{i_{1}} \cdots y_{i_{z}}$ in the equation $S(F)=-\mu^{-1} F$, we have

$$
-c_{\left(i_{s}\right)}^{\prime}=c_{1,\left(i_{s}\right)}(-1)^{z} \prod_{s>t} \lambda_{i_{s} i_{t}}^{-1}, \quad-c_{1,\left(i_{s}\right)}=c_{\left(i_{s}\right)}^{\prime}(-1)^{z} \prod_{a<b} \lambda_{i_{a} i_{b}}^{-1} .
$$

Since $c_{1,\left(i_{s}\right)}$ and $c_{\left(i_{s}\right)}^{\prime}$ are nonzero, the above two equations imply

$$
\prod_{s>t}\left(\lambda_{i_{s} i_{t}} \lambda_{i_{t} i_{s}}\right)=1
$$

or

$$
\prod_{\{s \neq t\} \subset\{1,2, \cdots, z\}}\left(\lambda_{i_{s} i_{t}} \lambda_{i_{t} i_{s}}\right)=1 .
$$

We know the monomial $y_{i_{1}} \cdots y_{i_{n}}$ contains $N_{i}$ copies of $y_{i}$ for all $i=1, \cdots, v$. Hence equation (I3.1.1) is in fact

$$
\prod_{i=1}^{v}\left(\lambda_{i i}\right)^{N_{i}\left(N_{i}-1\right)} \prod_{i<j}\left(\lambda_{i j} \lambda_{j i}\right)^{N_{i} N_{j}}=1 .
$$

There is a slight modification of Lemma 3.1. Suppose $\lambda_{11}$ is a primitive $p_{1}$-th root of unity for some $p_{1}>1$. Recycle most of the notation before Lemma 3.1. Let $L:=L\left(\Lambda, \mu, p_{1}\right)$ be the Hopf algebra generated as an algebra by the abelian group $G_{0}$ and $y_{1}, \cdots, y_{v}$ subject to the relations within $G_{0}$ and the following additional relations between $G_{0}$ and $\left\{y_{i}\right\}_{s=1}^{v}$ :

$$
\begin{aligned}
& y_{i} g=\lambda_{i}(g) g y_{i}+\tau_{i}(g) g\left(\mu_{i}-1\right), \text { for all } i \text { and all } g \in G_{0}, \\
& y_{1}^{p_{1}}=\beta\left(\mu_{1}^{p_{1}}-1\right), \quad \text { for some } \beta \in k .
\end{aligned}
$$

The coalgebra structure of $L$ is determined by

$$
\begin{aligned}
\Delta(g)=g \otimes g, & \epsilon(g)=1, \quad \text { for all } g \in G_{0}, \\
\Delta\left(y_{i}\right)=y_{i} \otimes 1+\mu_{i} \otimes y_{i}, & \epsilon\left(y_{i}\right)=0, \quad \text { for all } i=1, \cdots, v,
\end{aligned}
$$

and the antipode of $L$ is determined by

$$
\begin{aligned}
S(g) & =g^{-1}, \quad \text { for all } g \in G_{0}, \\
S\left(y_{i}\right) & =-\mu_{i}^{-1} y_{i}, \quad \text { for all } i=1, \cdots, v .
\end{aligned}
$$

Define $\Lambda_{M}:=\left(\lambda_{i j}\right)=\left(\lambda_{i}\left(\mu_{j}\right)\right)$. The total $y$-degree and the multi- $y$-degree are defined as before.

Lemma 3.2. Let $L:=L\left(\Lambda, \mu, p_{1}\right)$ be defined as above. Suppose either $\beta=0$ or $\lambda_{1}(g)^{p_{1}}=1$ for all $g \in G_{0}$.

(a) L has a k-linear basis

$$
\left\{g y_{i_{1}} y_{i_{2}} \cdots y_{i_{s}}\right\}
$$

where $g \in G_{0}, i_{1}, \cdots, i_{s} \in\{1, \cdots, v\}$ and there $i s$ no $u$ such that $i_{u}=$ $i_{u+1}=\cdots=i_{u+p_{1}-1}=1$. As a consequence, $L$ contains a free subalgebra $k\left\langle y_{1} y_{2}, y_{1} y_{2}^{2}\right\rangle$ and has exponential growth.

(b) The coradical of $L$ is $k G_{0}$. 
(c) If $F$ is a skew primitive element of total $y$-degree $z \geq 2$, then for any term of $F$ with multi-y-degree $\left(N_{1}, \cdots, N_{v}\right)$ and $\sum_{i} N_{i}=z$,

$$
\prod_{i=1}^{v}\left(\lambda_{i i}\right)^{N_{i}\left(N_{i}-1\right)} \prod_{i<j}\left(\lambda_{i j} \lambda_{j i}\right)^{N_{i} N_{j}}=1 .
$$

Proposition 3.3. Let $H$ be a Hopf algebra and $y_{1}, y_{2}$ be two skew primitive elements linearly independent in $H / C_{0}$. Suppose that

(i) there is a group-like element $x$ and $d_{1}, d_{2} \in \mathbb{Z}$ such that $\mu\left(y_{i}\right)=x^{d_{i}}$ for $i=1,2$, and

(ii) there are two scalars $q_{1}, q_{2} \in k^{\times}$such that $y_{i} x=q_{i} x y_{i}$ for $i=1,2$.

(a) If $x$ has infinite order and $H$ does not contain a free algebra of rank 2 , then

$$
q_{1}^{d_{1}\left(M_{1}\left(M_{1}-1\right)\right)+d_{2}\left(M_{1} M_{2}\right)} q_{2}^{d_{2}\left(M_{2}\left(M_{2}-1\right)\right)+d_{1}\left(M_{1} M_{2}\right)}=1
$$

for some integers $M_{1}, M_{2} \geq 0$ satisfying $M_{1}+M_{2} \geq 2$.

(b) Assume that one of the following holds:

(1) $q_{1}=q_{2}$ is not a root of unity and $d_{1} d_{2}>0$;

(2) $q_{1}^{d_{1}}=1$ and $q_{2}$ is not a root of unity and $d_{1} d_{2}>0$;

(3) $q_{1}^{d_{1}} \neq 1$ is a root of unity and $q_{2}$ is not a root of unity and $d_{1} d_{2}>0$;

(4) the group $\left\langle q_{1}, q_{2}\right\rangle \subset k^{\times}$is free abelian of rank $2, d_{1} d_{2} \neq 0$.

Then $H$ contains a free subalgebra of rank 2 . Consequently, $H$ has exponential growth.

Proof. (a) Let $\mu_{i}=x^{d_{i}}$ and $\lambda_{i j}=q_{i}^{d_{j}}$. Then $y_{i} \mu_{j}=\lambda_{i j} \mu_{j} y_{i}$ and $\gamma\left(y_{i}\right)=\lambda_{i i}$ for all $i, j \in\{1,2\}$.

Let $H_{0}$ be the Hopf subalgebra generated by $x, x^{-1}, y_{1}, y_{2}$. Let $G_{0}=\langle g\rangle \cong \mathbb{Z}$ and let $\lambda_{i}\left(g^{n}\right)=q_{i}^{n}$ for $i=1,2$ and all $n$. Let $\Lambda=\left\{\lambda_{1}, \lambda_{2}\right\}$ and $\mu=\{g, g\}$. Then there is a surjective Hopf algebra homomorphism $\phi: K:=K(\Lambda, \mu) \rightarrow H_{0}$ sending $g \mapsto x$ and $y_{i} \mapsto y_{i}$ for $i=1,2$, where we choose $\tau_{i}=0$. By Lemma 3.1(a), $K$ contains a free algebra of rank 2. If $H$ does not contain a free algebra of rank 2 , then $K \rightarrow H_{0}$ is not injective. By [Mo Theorem 5.3.1], there is a nonzero skew primitive element $F \in K$ such that $\phi(F)=0$. Since $\phi$ is injective on skew primitive elements of $y$-degree $\leq 1, F$ has total $y$-degree $z \geq 2$. By Lemma 3.1(c), for any term of $F$ with multi-y-degree $\left(M_{1}, M_{2}\right)$ and $M_{1}+M_{2}=z$, we have the following:

$$
\left(\lambda_{11}\right)^{M_{1}\left(M_{1}-1\right)}\left(\lambda_{22}\right)^{M_{2}\left(M_{2}-1\right)}\left(\lambda_{12} \lambda_{21}\right)^{M_{1} M_{2}}=1
$$

or, equivalently,

$$
\left(q_{1}^{d_{1}}\right)^{M_{1}\left(M_{1}-1\right)}\left(q_{2}^{d_{2}}\right)^{M_{2}\left(M_{2}-1\right)}\left(q_{1}^{d_{2}} q_{2}^{d_{1}}\right)^{M_{1} M_{2}}=1 .
$$

This can be simplified to

$$
q_{1}^{d_{1}\left(M_{1}\left(M_{1}-1\right)\right)+d_{2}\left(M_{1} M_{2}\right)} q_{2}^{d_{2}\left(M_{2}\left(M_{2}-1\right)\right)+d_{1}\left(M_{1} M_{2}\right)}=1,
$$

which is (13.3.1).

(b) Assume $H$ does not contain a free algebra of rank 2, and we will obtain a contradiction. If one of the hypotheses holds, then $x$ has infinite order in $G(H)$ by Lemma 2.2(d). Therefore we can apply part (a).

In case $(\mathrm{b})(1)$, 13.3 .1 implies that

$$
d_{1}\left(M_{1}\left(M_{1}-1\right)\right)+d_{2}\left(M_{1} M_{2}\right)+d_{2}\left(M_{2}\left(M_{2}-1\right)\right)+d_{1}\left(M_{1} M_{2}\right)=0 .
$$


This is impossible since $d_{1} d_{2}>0$ and $M_{1}+M_{2} \geq 2$. Therefore $H$ contains a free algebra of rank 2 .

A similar argument works for case (b)(4).

In case (b)(2), equation (I3.3.1) implies that $\left(q_{2}^{d_{2}\left(M_{2}\left(M_{2}-1\right)\right)+d_{1}\left(M_{1} M_{2}\right)}\right)^{d_{1}}=1$, or

$$
d_{2}\left(M_{2}\left(M_{2}-1\right)\right)+d_{1}\left(M_{1} M_{2}\right)=0
$$

because $q_{2}$ is not a root of unity. Since $d_{1} d_{2}>0$, the only solution is $M_{2}=0$ and $M_{1}=z \geq 2$. Thus we have $F=c y_{1}^{z}+l d t$ for some $c \in k^{\times}$. By Lemma 1.3 and the fact that $\lambda_{11}=q_{1}^{d_{1}}=1, F$ cannot be skew primitive for any $z \geq 2$. So case (b)(2) has been taken care of.

It remains to consider case (b)(3). Suppose $q_{1}^{d_{1}}$ is a primitive $p_{1}$-th root of unity. Then $y_{1}^{p_{1}}$ is a skew primitive element. If $y_{1}^{p_{1}} \notin C_{0}$, then $\left\{y_{1}^{p_{1}}, y_{2}\right\}$ is linearly independent in $H / C_{0}$. Note that if $\alpha y_{1}^{p_{1}}+\beta y_{2} \in C_{0}$, then $x^{-1}\left(\alpha y_{1}^{p_{1}}+\beta y_{2}\right) x \in C_{0}$, which would imply $y_{1}^{p_{1}}, y_{2} \in C_{0}$ because $q_{1}^{p_{1}} \neq q_{2}$. The assertion follows from case (b)(2) applied to $\left\{y_{1}^{p_{1}}, y_{2}\right\}$. If $y_{1}^{p_{1}} \in C_{0}$, then $y_{1}^{p_{1}}=\beta\left(\mu_{1}^{p_{1}}-1\right)$ for some $\beta$. Replacing $K$ by $L$ in the above argument, (I3.3.1) holds again.

Since $q_{1}$ is a root of unity, we have

$$
\left(q_{2}^{p}\right)^{d_{2}\left(M_{2}\left(M_{2}-1\right)\right)+d_{1}\left(M_{1} M_{2}\right)}=1
$$

for some $p>1$. Since $q_{2}$ is not a root of unity,

$$
d_{2}\left(M_{2}\left(M_{2}-1\right)\right)+d_{1}\left(M_{1} M_{2}\right)=0 .
$$

Since $d_{1} d_{2}>0$, the only solution is $M_{2}=0$ and $M_{1}=z \geq 2$. Now $F=c y_{1}^{z}+l d t$, where $c \in k^{\times}$and $z<p_{1}$. By Lemma 1.3, $F$ cannot be skew primitive. This is a contradiction.

Corollary 3.4. Suppose $H$ has subexponential growth. Let y be a skew primitive element not in $C_{0}$ such that $\gamma(y)$ is defined and is not a root of unity. Let $G_{0}$ be a finitely generated abelian subgroup of $G(H)$ containing $\mu(y)$ (which has infinite order automatically). Then $V:=k(\mu(y)-1)+\sum_{g \in G_{0}} k\left(g^{-1} y g\right)$ is 2-dimensional. As a consequence, there is a group representation $\lambda: G_{0} \rightarrow k$ such that

$$
g^{-1} y^{\prime} g=\lambda(g) y^{\prime}
$$

for all $g \in G_{0}$, where $y^{\prime}=y+\alpha(\mu(y)-1)$ for some $\alpha \in k$.

Proof. Since $\gamma(y)$ is not a root of unity, we may assume that $y \mu(y)=\gamma(y) \mu(y) y$ after replacing $y$ by $y+\alpha(\mu(y)-1)$ for some $\alpha \in k$. Let $g \in G_{0}$. Let $y_{1}=y$ and $y_{2}=g^{-1} y g$. Then $\gamma\left(y_{1}\right)=\gamma\left(y_{2}\right)$, and it is not a root of unity. By Proposition 3.3. (b)(1), $y_{1}$ and $y_{2}$ are not linearly independent in $H / C_{0}$. The assertion that $\operatorname{dim} V=2$ follows by applying Lemma 1.6. The consequence follows from Lemma $2.2(\mathrm{ci})$.

Proof of Proposition 0.5. We prove the assertion by contradiction. So we assume that $H$ has subexponential growth.

Pick a pair of skew primitive elements $\left(y_{1}, y_{2}\right)$ such that the subgroup of $\langle\Gamma\rangle$ generated by $\left\{\gamma\left(y_{1}\right), \gamma\left(y_{2}\right)\right\}$ has rank 2 . Let $\lambda_{i i}=\gamma\left(y_{i}\right)$ and $g_{i}=\mu\left(y_{i}\right)$ for $i=1,2$. Since $\langle W\rangle$ is abelian, the subgroup $G_{0}:=\left\langle g_{1}, g_{2}\right\rangle$ is abelian. By Corollary 3.4. we may further assume that, for $i=1,2$ and $j=1,2, g_{j}^{-1} y_{i} g_{j}=\lambda_{i j} y_{i}$ for some 
$\lambda_{i j} \in k^{\times}$. Since $H$ does not contain a free subalgebra of rank 2 , the proofs of Proposition 3.3 and (13.3.1) show that

$$
\left(\lambda_{11}\right)^{M_{1}\left(M_{1}-1\right)}\left(\lambda_{22}\right)^{M_{2}\left(M_{2}-1\right)}\left(\lambda_{12} \lambda_{21}\right)^{M_{1} M_{2}}=1
$$

for some nonnegative $M_{1}, M_{2}$ with $M_{1}+M_{2} \geq 2$.

Since $G_{0}$ is a finitely generated subgroup of $\langle W\rangle$ and since $\langle W\rangle$ is abelian and torsionfree of rank $1, G_{0}$ is isomorphic to $\mathbb{Z}$. Therefore there is an $x \in G_{0}$ such that $g_{1}=x^{a}$ and $g_{2}=x^{b}$ for some nonzero integers $a, b$. Consequently, $g_{2}^{a}=g_{1}^{b}$. Thus the equation $g_{j}^{-1} y_{i} g_{j}=\lambda_{i j} y_{i}$ implies that $\lambda_{i 2}^{a}=\lambda_{i 1}^{b}$ for all $i=1,2$. Then (I3.4.1) implies that

$$
\left(\lambda_{11}\right)^{a b M_{1}\left(M_{1}-1\right)}\left(\lambda_{22}\right)^{a b M_{2}\left(M_{2}-1\right)}\left(\lambda_{11}\right)^{b^{2} M_{1} M_{2}}\left(\lambda_{22}\right)^{a^{2} M_{1} M_{2}}=1 .
$$

Since the rank of $\left\langle\lambda_{11}, \lambda_{22}\right\rangle$ is 2 , we have

$$
a b M_{1}\left(M_{1}-1\right)+b^{2} M_{1} M_{2}=a b M_{2}\left(M_{2}-1\right)+a^{2} M_{1} M_{2}=0
$$

or

$$
a\left(M_{1}-1\right)+b M_{2}=b\left(M_{2}-1\right)+a M_{1}=0 .
$$

Since $a, b$ are nonzero, this means that $\left(M_{1}-1\right)\left(M_{2}-1\right)-M_{1} M_{2}=0$. This is impossible when $M_{1}+M_{2} \geq 2$, which yields a contradiction.

The rest of this section is devoted to the proof of Theorem 0.4 . The next definition was given in the introduction, but perhaps it should be reviewed here. Let $y$ be a $(1, g)$-primitive element in a Hopf algebra $H$. Let $T_{g^{-1}}$ denote the inverse conjugation by $g$, namely, $T_{g^{-1}}: a \rightarrow g^{-1} a g$.

Definition 3.5. Let $y$ be a $(1, g)$-primitive element of $H$ not in $C_{0}$. A nonzero scalar $\lambda$ is called the commutator of $y$ of level $n$ if $\left(T_{g^{-1}}-\lambda I d_{H}\right)^{n}(y) \in C_{0}$ and $\left(T_{g^{-1}}-\lambda I d_{H}\right)^{n-1}(y) \notin C_{0}$. In this case we write $\gamma(y)=\lambda$. When $n=1, \gamma(y)$ is the commutator of $y$ defined as in (10.3.2) or equivalently in (10.3.3).

In general, the commutator of $y$ may not exist. We also need a generalization of Definition 3.5. Recall that $W_{\times}$is the subset of $W$ consisting of weights $\mu(y)$ such that the commutator of $y$ is either 1 or not a root of unity. Throughout the rest of the section let $G_{0}$ be the subgroup $\left\langle W_{\times}\right\rangle$and suppose that $G_{0}$ is abelian. A 1dimensional representation of $G_{0}$ is equivalent to a multiplicative map $\lambda: G_{0} \rightarrow k^{\times}$. Let $G_{0}^{*}$ denote the set of 1 -dimensional representations of $G_{0}$, which is also called the character group of $G_{0}$.

Definition 3.6. Let $y$ be a skew primitive element in $H \backslash C_{0}$ and let $\lambda \in G_{0}^{*}$. We say $\lambda$ is the generalized commutator of $y$ of level $n$ if there is an $n$ such that $\left(T_{g^{-1}}-\lambda(g) I d_{H}\right)^{n}(y) \in C_{0}$ for all $g \in G_{0}$ and $\left(T_{g^{-1}}-\lambda(g) I d_{H}\right)^{n-1}(y) \notin C_{0}$ for some $g \in G_{0}$. If $n=1, \lambda$ is called the generalized commutator of $y$.

Lemma 3.7. In parts (a) and (c) suppose that GKdim $H<\infty$.

(a) Every skew primitive element $y \in H$ is a linear combination of skew primitives with commutator of finite level and weight $\mu(y)$.

(b) Suppose $V$ is a conjugation $G_{0}$-stable finite dimensional subspace spanned by skew primitives with their weights in $G_{0}$. Then every element $y \in V$ is a linear combination of skew primitives with generalized commutator of finite level. 
(c) Every skew primitive $y \in H$ with commutator of level 1 such that $\gamma(y) \in \Gamma \backslash$ $\Gamma_{\sqrt{ }}$ is a linear combination of skew primitives with generalized commutator of finite level. Further, each nonzero summand of the linear combination has weight commutator $\omega(y)$.

Proof. (a) If $y \in C_{0}$, then $y=\alpha(\mu(y)-1)$ for some $\alpha \in k$ [Lemma 1.6] and the commutator of $y$ has level 0 by definition.

If $y \notin C_{0}$, then, by Lemma [2.5, $V:=\sum_{n \in \mathbb{Z}} k\left(g^{-n} y g^{n}\right)+k(g-1)$ is finite dimensional over $k$, where $g=\mu(y)$. Then $T_{g^{-1}}$ acts on $V$ as an invertible linear map. Pick a basis of $V$ so that the presentation of $T_{g^{-1}}$ with respect to the basis is in the Jordon canonical form. Then each basis element is a skew primitive element with commutator of finite level. The assertion follows.

(b) Write $V=\bigoplus_{j=1}^{m} V_{j}$, where each $V_{j}$ is spanned by skew primitives with weight $g_{j}$ for distinct group-like elements $g_{1}, \cdots, g_{m} \in G_{0}$. Since $G_{0}$ is abelian, each $V_{j}$ is conjugation $G_{0}$-stable. Passing from $V$ to $V_{j}$ we may assume that each element in $V$ is a skew primitive of weight $g$.

For every $h \in G_{0}, T_{h^{-1}}$ acts on $V$ as an invertible linear map. It is clear that $V$ is a finite dimensional $k G_{0}$-module. Since $k G_{0}$ is commutative, every finite dimensional simple $G_{0}$-module is 1-dimensional and $\operatorname{Ext}_{k G_{0}}^{1}\left(S, S^{\prime}\right)=0$ if $S$ and $S^{\prime}$ are distinct simple modules over $k G_{0}$. Then $V$ is a finite direct sum of submodules $V_{i}$ so that the support of each $V_{i}$ is a single closed point of Spec $k G_{0}$. This closed point corresponds to a 1-dimensional $G_{0}$-representation $\lambda_{i}$. Fix any $i$, every element in $V_{i}$ has a generalized commutator $\lambda_{i}$ with level no more than $\operatorname{dim} V_{i}$.

(c) Let $V$ be the vector space spanned by all skew primitive elements $z$ with commutator of level 1 such that $\omega(z)=\omega(y)$. Pick any finite set $\left\{z_{1}, \cdots, z_{w}\right\}$ which is linearly independent in $V /(V \cap k(\mu(y)-1))$. Then (I1.2.1)-(I1.2.3) hold for $D=C_{0}$ and $A=k\left[\mu(y)^{ \pm 1}\right]$. By Theorem [1.5(b), $w \leq \operatorname{GKdim} H$. Thus $V$ is finite dimensional. Clearly, $T_{g^{-1}}$ stabilizes $V$ for all $g \in G_{0}$. The first assertion follows from part (b). The final assertion is clear since every element in $V$ has weight commutator equal $\omega(y)$.

We need to introduce some conventions. Let $\mu \in W$. Define $P_{\mu}$ to be the $k$ linear space spanned by all skew primitives $y \in H$ with $\mu(y)=\mu$. Let $\gamma$ be a nonzero scalar and let $P_{\mu, \gamma, n}$ be the $k$-linear space spanned by all skew primitives $y \in P_{\mu}$ with commutator $\gamma$ of level no more than $n$. Let $P_{\mu, \gamma, *}=\sum_{n \geq 0} P_{\mu, \gamma, n}$, $P_{*, *, n}=\sum_{\mu, \gamma} P_{\mu, \gamma, n}$ and $P_{*, *, *}=\sum_{\mu, \gamma, n} P_{\mu, \gamma, n}$.

Given any $\mu \in W$ and $\lambda \in G_{0}^{*}$, let $P_{\mu, \lambda, n}$ be the $k$-linear space spanned by all skew primitives $y \in H$ with generalized commutator $\lambda$ of level no more than $n$ and $\mu(y)=\mu$. Let $P_{\mu, \lambda, *}=\sum_{n \geq 0} P_{\mu, \lambda, n}, P_{*, G *, n}=\sum_{\mu, \lambda} P_{\mu, \lambda, n}$ and $P_{*, G *, *}=$ $\sum_{\mu, \lambda, n} P_{\mu, \lambda, n}$

Lemma 3.7(a) says that $P_{*, *, *}$ contains all skew primitive elements, and Lemma 3.7(c) says that $P_{\mu, \gamma, 1}$ is a subspace of $P_{*, G *, *}$ when $\gamma \in k^{\times}$and $\gamma$ is 1 or not a root of unity.

Lemma 3.8. Retain the above notation. Suppose that $H$ does not contain a free subalgebra of rank 2 .

(a) If $P_{\mu, \gamma, 1} \subset k(\mu-1)$, then $P_{\mu, \gamma, *} \subset k(\mu-1)$.

(b) If $P_{\mu, \gamma, 1} \not \subset k(\mu-1)$ and $\gamma$ is not a root of unity, then $P_{\mu, \gamma_{0}, *} \subset k(\mu-1)$ for every root of unity $\gamma_{0}$.

(c) If $\gamma$ is not a root of unity, then $P_{\mu, \gamma, 1} / k(\mu-1)$ has dimension at most 1 . 
(d) Suppose $\gamma_{1}$ and $\gamma_{2}$ are two distinct scalars, neither of which is a root of unity. If $P_{\mu, \gamma_{i}, 1} \not \subset k(\mu-1)$ for $i=1,2$, then $\gamma_{1}^{N} \gamma_{2}^{M}=1$ for some positive integers $N, M$. Further, there is no $\gamma_{3} \in k^{\times} \backslash\left\{\gamma_{1}, \gamma_{2}\right\}$ such that $P_{\mu, \gamma_{3}, 1} \not \subset$ $k(\mu-1)$.

(e) Suppose that GKdim $H<\infty$. If $\gamma$ is not a root of unity, then $P_{\mu, \gamma, *}=$ $P_{\mu, \gamma, 1} \subset P_{*, G *, *}$.

Proof. (a) This is clear by induction.

(b) First assume that $\gamma_{0}$ is not 1. Pick $y_{2} \in P_{\mu, \gamma, 1} \backslash C_{0}$. If $P_{\mu, \gamma_{0}, *} \not \subset k(\mu-1)$ for a root of unity $\gamma_{0}$, by part (a), there is a $y_{1} \in P_{\mu, \gamma_{0}, 1} \backslash C_{0}$. Since $\gamma_{0}$ and $\gamma$ are not 1 , after adding a suitable term $\alpha\left(\mu\left(y_{i}\right)-1\right)$ to $y_{i}$, the hypothesis in Proposition 3.3(ii) holds. Then we are in the situation of Proposition 3.3.(b)(2), (b)(3) (where $d_{1}=d_{2}=1$ ). By Proposition 3.3. $H$ contains a free subalgebra of rank 2, a contradiction.

Second, assume that $\gamma_{0}=1$. For the statement we only need to consider the Hopf subalgebra generated by group-like and skew primitive elements. So we may assume that $H$ is pointed. Passing to the associated graded Hopf algebra of $H$ with respect to its coradical filtration, one can assume the hypothesis in Proposition 3.3(ii) holds. So the above proof works.

(c) This follows from Proposition 3.3(b)(1).

(d) By the proof of Proposition 3.3, see (I3.3.1),

$$
q_{1}^{d_{1}\left(M_{1}\left(M_{1}-1\right)\right)+d_{2}\left(M_{1} M_{2}\right)} q_{2}^{d_{2}\left(M_{2}\left(M_{2}-1\right)\right)+d_{1}\left(M_{1} M_{2}\right)}=1,
$$

where $M_{1}$ and $M_{2}$ are nonnegative integers with $M_{1}+M_{2} \geq 2$. Note that $\gamma_{i}=q_{i}$ and $d_{1}=d_{2}=1$ in this case. Let $N=M_{1}\left(M_{1}-1\right)+M_{1} M_{2}$ and $M=M_{2}\left(M_{2}-\right.$ 1) $+M_{1} M_{2}$. Then $N$ and $M$ are nonnegative and $N+M \geq 2$. Since $\gamma_{1}$ and $\gamma_{2}$ are not roots of unity, both $N$ and $M$ must be positive.

If such a $\gamma_{3}$ exists, by part (b) it is not a root of unity. By the above assertion we have positive integers $A, B, C, D$ such that $\gamma_{1}^{A} \gamma_{3}^{B}=1$ and $\gamma_{2}^{C} \gamma_{3}^{D}=1$. Then

$$
1=\left(\gamma_{1}^{N} \gamma_{2}^{M}\right)^{A C}=\gamma_{1}^{A C N} \gamma_{2}^{C A M}=\gamma_{3}^{-B C N-D A M},
$$

which contradicts the fact that $\gamma_{3}$ is not a root of unity.

(e) If $P_{\mu, \gamma, *} \neq P_{\mu, \gamma, 1}$, pick a skew primitive $y_{2} \in P_{\mu, \gamma, 2}$. This means that $\mu^{-1} y_{2} \mu-\gamma y_{2}=y_{1} \in P_{\mu, \gamma, 1} \backslash k(\mu-1)$. Consider the Hopf subalgebra $K$ generated as an algebra by $y_{2}, y_{1}, \mu, \mu^{-1}$. By possibly adding a term $\beta(\mu-1)$ to $y_{1}$ for some $\beta \in k$ and adding a term $\alpha(\mu-1)$ to $y_{2}$ for some $\alpha \in k$, we can assume that $y_{1} \mu=\gamma \mu y_{1}$ and $y_{2} \mu=\gamma \mu y_{2}+\mu y_{1}$.

It remains to show that $K$ contains a free subalgebra of rank 2. Define a filtration $F_{i}$ of $K$ inductively as follows:

$$
\begin{aligned}
& F_{0}=C_{0}(K)=k\left[\mu^{ \pm 1}\right], \\
& F_{1}=F_{0}+F_{0} y_{1}=F_{0} y_{1}+F_{0}, \\
& F_{2}=F_{0}+F_{0} y_{1}+F_{0} y_{1}^{2}+F_{0} y_{2}=F_{0}+y_{1} F_{0}+y_{1}^{2} F_{0}+y_{2} F_{0}, \\
& F_{n}=\sum_{i=1}^{n-1} F_{i} F_{n-i} \text { for all } n \geq 3 .
\end{aligned}
$$

It is easy to check that $F$ is a Hopf algebra filtration and $\operatorname{gr}_{F} K$ is a Hopf

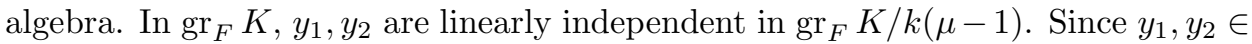
$P_{\mu, \gamma, 1}(K)$, by part (c), $\operatorname{gr}_{F} K$ contains a free subalgebra of rank 2 . Therefore $K$ contains a free subalgebra of rank 2 . This yields a contradiction. Therefore the first assertion (namely, the first equation) follows. Finally, by Lemma 3.7(c), $P_{\mu, \gamma, 1} \subset P_{*, G *, *}$. 
Remark 3.9. Suppose GKdim $H<\infty$. By Lemmas 2.1(b) and 3.7(a), $W \backslash W_{\sqrt{ }} \subseteq$ $W_{\times}$. In practice it often happens that $W \backslash W_{\sqrt{ }}=W_{\times}$.

Now we are ready to prove the Third Lower Bound Theorem. Let $Y_{*}$ be the $k$-linear vector space spanned by all skew primitive elements $y$ with commutator of finite level such that $\gamma(y) \in \Gamma \backslash \Gamma_{\sqrt{ }}$.

Theorem 3.10. Suppose $G_{0}$ is abelian. Then

$$
\operatorname{GKdim} H \geq \operatorname{GKdim} C_{0}+\operatorname{dim} Y_{*} /\left(Y_{*} \cap C_{0}\right) .
$$

Proof. Nothing needs to be proved if $\operatorname{GKdim} H=\infty$, so we assume GKdim $H<\infty$.

Let

$$
Y_{n}=\sum_{\mu \in W_{\times}, \gamma \in \Gamma \backslash \Gamma_{\mathcal{V}}} P_{\mu, \gamma, n} \text { and } Y_{G n}=\sum_{\mu \in W_{\times}, \lambda \in G_{0}^{*}, \gamma:=\lambda(\mu) \in \Gamma \backslash \Gamma_{\mathcal{V}^{-}}} P_{\mu, \lambda, n}
$$

for all $n \geq 1$. Then $Y_{*}=\sum_{n} Y_{n}$. Let $Y_{G *}=\sum_{n} Y_{G n}$. We prove the following claim by induction:

Claim A.

$$
\operatorname{GKdim} H \geq \operatorname{GKdim} C_{0}+\operatorname{dim} Y_{G n} /\left(Y_{G n} \cap C_{0}\right)
$$

for all $n \geq 1$. When $n=1$, let $\left\{y_{i}\right\}$ be a basis of $Y_{G 1} /\left(Y_{G 1} \cap C_{0}\right)$ such that each $y_{i}$ is in $P_{\mu_{i}, \lambda_{i}, 1}$ for some $\mu_{i}, \lambda_{i}$. Then we have

$$
\mu_{j}^{-1} y_{i} \mu_{j}=\lambda_{i}\left(\mu_{j}\right) y_{i}+\tau_{i j}\left(\mu_{i}-1\right)
$$

where $\lambda_{i}\left(\mu_{i}\right)=\gamma\left(y_{i}\right)$ is either 1 or not a root of unity and where $\tau_{i j} \in k$. By Theorem 1.5 (with $D=C_{0}$ and $A=k G_{0}$ ),

$$
\operatorname{GKdim} H \geq \operatorname{GKdim} C_{0}+\#\left\{y_{i}\right\}=\operatorname{GKdim} C_{0}+\operatorname{dim} Y_{G 1} /\left(Y_{G 1} \cap C_{0}\right),
$$

which proves Claim A for $n=1$.

Now assume that Claim A holds for $n$. Without loss of generality we may assume that $H$ is generated as an algebra by $C_{0}$ and all skew primitive elements of $H$. Define a filtration $F_{i}$ of $H$ as follows:

$F_{0}=C_{0}$,

$F_{1}=F_{0}+F_{0} Y_{G 1}=F_{0}+Y_{G 1} F_{0}$,

$F_{2}=F_{1}^{2}+F_{0} Z$, where $Z$ is the $k$-linear span of all skew primitive elements of $H$, and

$$
F_{m}=\sum_{i=1}^{m-1} F_{i} F_{m-i} \text { for all } m \geq 3 \text {. }
$$

Then $\left\{F_{m}\right\}$ is a Hopf algebra filtration of $H$. Let $K$ be the associated graded Hopf algebra $\operatorname{gr}_{F} H$. Note that if $y \in P_{\mu, \lambda, 2} \subset Y_{G 2}(H)$, then $y \in F_{2}$ and the associated element in $K$ is gr $y \in F_{2} / F_{1}$, and $g^{-1}$ (gr $\left.y\right) g=\lambda(g)$ (gr $\left.y\right)$ for all $g \in G_{0}$ (or, when trivially $y \in F_{1}$, we have gr $y \in F_{1} / F_{0}$ or gr $y \in F_{0}$ ). Thus gr $y \in Y_{G 1}(K)$ (which is easy to see when $y \in F_{1}$ ). By induction one sees that

$$
Y_{G n}(K) \supseteq\left\{\operatorname{gr} y \mid y \in Y_{G(n+1)}(H)\right\}=: \operatorname{gr} Y_{G(n+1)}(H)
$$

for all $n$. Applying the induction hypothesis to $K$,

$$
\operatorname{GKdim} K \geq \operatorname{GKdim} C_{0}+\operatorname{dim} Y_{G n}(K) /\left(Y_{G n}(K) \cap C_{0}\right) .
$$

By [KL, Lemma 6.5], GKdim $H \geq \operatorname{GKdim} K$. It is clear that

$$
\operatorname{dim} Y_{G(n+1)}(H) /\left(Y_{G(n+1)}(H) \cap C_{0}\right)=\operatorname{dimgr} Y_{G(n+1)}(H) /\left(\operatorname{gr} Y_{G(n+1)}(H) \cap C_{0}\right) .
$$


Therefore,

$$
\begin{aligned}
\operatorname{GKdim} H & \geq \operatorname{GKdim} K \geq \operatorname{GKdim} C_{0}+\operatorname{dim} Y_{G n}(K) /\left(Y_{G n}(K) \cap C_{0}\right) \\
& \geq \operatorname{GKdim} C_{0}+\operatorname{dim} \operatorname{gr} Y_{G(n+1)}(H) /\left(\operatorname{gr} Y_{G(n+1)}(H) \cap C_{0}\right) \\
& =\operatorname{GKdim} C_{0}+\operatorname{dim} Y_{G(n+1)}(H) /\left(Y_{G(n+1)}(H) \cap C_{0}\right),
\end{aligned}
$$

which finishes the induction step. Therefore we proved Claim A.

When $n$ goes to infinity, we have

$$
\operatorname{GKdim} H \geq \operatorname{GKdim} C_{0}+\operatorname{dim} Y_{G *}(H) /\left(Y_{G *}(H) \cap C_{0}\right) .
$$

Next we prove the following claim by induction:

Claim B.

$$
\operatorname{GKdim} H \geq \operatorname{GKdim} C_{0}+\operatorname{dim} Y_{n} /\left(Y_{n} \cap C_{0}\right) .
$$

When $n=1$, this follows from (I3.10.1) since $Y_{1}(H) \subset Y_{G *}(H)$ by Lemma 3.7(c). Note that $Y_{1}$ is $G_{0}$-stable. Using an argument similar to the proof of Claim A by passing to the associated graded Hopf algebra $\operatorname{gr}_{F} H$ (and replacing $Y_{G n}$ by $Y_{n}$ ), one sees that Claim B holds. When $n$ goes to infinity, we have

$$
\operatorname{GKdim} H \geq \operatorname{GKdim} C_{0}+\operatorname{dim} Y_{*}(H) /\left(Y_{*}(H) \cap C_{0}\right) .
$$

The proof of Theorem 3.10 also shows the following.

Corollary 3.11. Suppose $G_{0}$ is abelian and GKdim $H<\infty$. Let $\mu \in W$ and suppose that $\gamma \in k^{\times}$is not a root of unity. Then

(a) $P_{\mu, 1, *}$ is finite dimensional.

(b) $P_{\mu, 1, *}$ and $P_{\mu, \gamma, *}$ are subspaces of $P_{*, G *, *}$.

(c) $Y_{*}=Y_{G *}$.

By Lemma 3.8(c), (e), $P_{\mu, \gamma, *}$ is finite dimensional.

Proof of Corollary [3.11, (a) By Theorem 3.10, $Y_{*} /\left(Y_{*} \cap C_{0}\right)$ is finite dimensional. Since $P_{\mu, 1, *} \subset Y_{*}$ and $P_{\mu, 1, *} \cap C_{0}=k(\mu-1)$, we have

$$
\operatorname{dim} P_{\mu, 1, *} / k(\mu-1) \leq \operatorname{dim} Y_{*} /\left(Y_{*} \cap C_{0}\right)<\infty,
$$

which implies that $P_{\mu, 1, *}$ is finite dimensional.

(b) By Lemma 3.8(e), $P_{\mu, \gamma, *}$ is a subspace of $P_{*, G *, *}$.

By part (a) $P_{\mu, 1, *}$ is finite dimensional. It is clear that $P_{\mu, 1, *}$ is $G_{0^{-}}$-stable. By Lemma 3.7(b), (c) $P_{\mu, 1, *}$ is a subspace of $P_{*, G *, *}$.

(c) As a consequence of part (b), $P_{\mu, \lambda, *}=P_{\mu, \lambda(\mu), *}$ when $\lambda(\mu)$ is either 1 or not a root of unity. The assertion follows.

Theorem 0.4 is an immediate consequence of Theorem 3.10 .

Proof of Theorem 0.4. Without loss of generality we assume that GKdim $H<\infty$. First we claim that $Y_{*} \cap\left(Y_{\sqrt{ }}+C_{0}\right) \subset C_{0}$. Suppose $y=z+c$ is in $Y_{*} \cap\left(Y_{\sqrt{ }}+C_{0}\right)$, where $y \in Y_{*} \backslash C_{0}$ and $z \in Y_{\sqrt{ }}$ and $c \in C_{0}$. It is easily reduced to the case when $\mu(y)=\mu(z)=g$ and $c=\alpha(g-1)$ for some $\alpha \in k$. Then $y \in Y_{\sqrt{ }}$, a contradiction. Therefore the claim holds. 
By Lemma 3.7(a), every skew primitive element is a linear combination of skew primitives with commutator of finite level. This says that $Z=Y_{*}+Y_{\sqrt{ }}+C_{0}$. Since $Y_{*} \cap\left(Y_{\sqrt{ }}+C_{0}\right) \subset C_{0}$, we have

$$
Z /\left(C_{0}+Y_{\sqrt{ }}\right) \cong Y_{*} / Y_{*} \cap\left(C_{0}+Y_{\mathcal{V}^{-}}\right)=Y_{*} /\left(Y_{*} \cap C_{0}\right) .
$$

The assertion follows by Theorem 3.10 .

Another way of proving Theorem 0.3 (with a slightly stronger hypothesis that $\left\langle W_{\times}\right\rangle$is abelian) is by using Theorem 0.4 and the following lemma, which is due to an anonymous referee.

Lemma 3.12. Suppose GKdim $H<\infty$. Then

$$
\operatorname{dim} Z /\left(C_{0}+Y_{\sqrt{ }}\right) \geq \#\left(W \backslash W_{\sqrt{ }}\right) .
$$

Proof. For any $w \leq \#\left(W \backslash W_{\sqrt{ }}\right)$, take distinct elements $x_{1}, \cdots, x_{w} \in W \backslash W_{\sqrt{ }}$; we need to prove that $\operatorname{dim} Z /\left(C_{0}+Y_{\sqrt{ }}\right) \geq w$.

For each $i$, pick a skew primitive element $z_{i} \in H \backslash C_{0}$ such that $x_{i}=\mu\left(z_{i}\right)$. Lemma 2.5 shows how to get a suitable $z_{i}$ such that $\gamma\left(z_{i}\right)$ is defined. Since $\mu\left(z_{i}\right)=$ $x_{i} \notin W_{\sqrt{ }}$, Lemma 2.1(b) says that $\gamma\left(z_{i}\right)$ is either 1 or not a root of unity.

It suffices to show that $z_{1}, \cdots, z_{w}$ are linearly independent in $Z /\left(C_{0}+Y_{\sqrt{ }}\right)$, so it is enough to show that $z_{1}, \cdots, z_{w}, y$ are linearly independent in $Z / C_{0}$ for any $y \in Y_{\sqrt{ }} \backslash C_{0}$. We can arrange $y=y_{1}+\cdots+y_{v}$ for some skew primitive elements $y_{j} \notin C_{0}$, where $y_{j}$ has a commutator $\gamma\left(y_{j}\right)$ of finite level which is a nontrivial root of unity and where the pairs $\left(\mu\left(y_{j}\right), \gamma\left(y_{j}\right)\right)$ are distinct. The pairs $\left(\mu\left(z_{i}\right), \gamma\left(z_{i}\right)\right)$ are already distinct, and $\left(\mu\left(z_{i}\right), \gamma\left(z_{i}\right)\right) \neq\left(\mu\left(y_{j}\right), \gamma\left(y_{j}\right)\right)$ for all $i, j$ because $\gamma\left(z_{i}\right)$ is either 1 or not a root of unity.

An improved version of Lemma 2.3 for skew primitive elements with commutators of finite level says that $z_{1}, \cdots, z_{w}, y_{1}, \cdots, y_{v}$ are linearly independent in $Z / C_{0}$. Consequently, $z_{1}, \cdots, z_{w}, y$ are linearly independent in $Z / C_{0}$, as desired.

Finally, we end with a simple example in which $Z=Y_{\sqrt{-}^{-}}+C_{0}$.

Example 3.13. Let $H$ be generated as an algebra by $x$ and $\left\{y_{i}\right\}_{i=1}^{\infty}$ subject to the relations

$$
\begin{aligned}
x^{2} & =1, \\
y_{i}^{2} & =0, \\
y_{i} y_{j}+y_{j} y_{i} & =0, \\
x y_{i}+y_{i} x & =0
\end{aligned}
$$

for all $i, j \in \mathbb{N}$. The coalgebra structure and the antipode of $H$ are determined by

$$
\begin{aligned}
\Delta(x)=x \otimes x, & \Delta\left(y_{i}\right)=y_{i} \otimes 1+x \otimes y_{i}, \\
\epsilon(x)=1, & \epsilon\left(y_{i}\right)=0, \\
S(x)=x, & S\left(y_{i}\right)=-x y_{i}=y_{i} x
\end{aligned}
$$

for all $i$. It is easy to check the following:

(a) $\operatorname{GKdim} H=0$,

(b) $\Omega=\{(x,-1)\}=\Omega_{\sqrt{ }}$,

(c) $Z=Y_{\sqrt{ }}+C_{0}$ and $\operatorname{dim} Y_{\sqrt{ }}=\infty$,

(d) $P_{x,-1, *}=P_{*,-1,1}=Y_{\sqrt{ }}$. 


\section{ACKNOWLEDGMENTS}

The authors thank Ken Goodearl and Ken Brown for their valuable comments and thank the referee for a careful reading, many comments, corrections, suggestions and Lemma 3.12. A part of this research was done when the second author was visiting Fudan University in the Fall of 2009 and the Spring of 2010. The first author was supported by the National Natural Science Foundation of China (No. 10671016 and 11171183) and the Shandong Provincial Natural Science Foundation of China (No. ZR2011AM013). The second and third authors were supported by the US National Science Foundation (NSF grant No. DMS 0855743).

\section{REFERENCES}

[AA] N. Andruskiewitsch and I.E. Angiono, On Nichols algebras with generic braiding, pp. 47-64 in "Modules and comodules" (Porto, Portugal, 2006), edited by T. Brzeziński et al., Birkhäuser, Basel, 2008. MR2742620 (2012b:16079)

[AS1] N. Andruskiewitsch and H.-J. Schneider, Pointed Hopf Algebras, in: Recent developments in Hopf algebra Theory, MSRI Publications 43 (2002), 168, Cambridge Univ. Press. MR 1913436 (2003e:16043)

[AS2] , A characterization of quantum groups, J. Reine Angew. Math. 577 (2004), 81104. MR2108213 (2005i:16083)

[Be] G.M. Bergman, The diamond lemma for ring theory, Adv. in Math. 29 (2) (1978), 178218. MR506890 (81b:16001)

[Br1] K. A. Brown, Noetherian Hopf algebras. Turkish J. Math. 31 (2007), suppl., 7-23. MR2368080 (2008j:16106)

[Br2] - Representation theory of Noetherian Hopf algebras satisfying a polynomial identity, in Trends in the Representation Theory of Finite Dimensional Algebras (Seattle 1997), (E.L. Green and B. Huisgen-Zimmermann, eds.), Contemp. Math. 229 (1998), 49-79. MR1676211 (99m:16056)

[BG1] K. A. Brown and K. R. Goodearl, Homological aspects of Noetherian PI Hopf algebras and irreducible modules of maximal dimension, J. Algebra 198 (1997), 240-265. MR 1482982 (99c:16036)

[BG2] Lectures on Algebraic Quantum Groups, Birkhäuser, 2002. MR.1898492 (2003f:16067)

[BZ] K.A. Brown and J.J. Zhang, Prime regular Hopf algebras of GK-dimension one, Proc. London Math. Soc. (3) 101 (2010) 260-302. MR2661247 (2011j:16063)

[GZ1] K.R. Goodearl and J.J. Zhang, Homological properties of quantized coordinate rings of semisimple groups, Proc. Lond. Math. Soc. (3) 94 (2007), no. 3, 647-671. MR2325315 (2008e:20077)

[GZ2] , Noetherian Hopf algebra domains of Gelfand-Kirillov dimension two, J. of Algebra 324 (2010), Special Issue in Honor of Susan Montgomery, 3131-3168. MR2732991 (2012d:16098)

[Gr] M. Gromov, Groups of polynomial growth and expanding maps. Inst. Hautes Études Sci. Publ. Math. No. 53 (1981), 53-73. MR623534(83b:53041)

[Kh] V.K. Kharchenko, A quantum analogue of the Poincaré-Birkhoff-Witt theorem (Russian. Russian summary), Algebra Log. 38 (1999), no. 4, 476-507, 509; translation in Algebra and Logic 38 (1999), no. 4, 259-276. MR.1763385 (2001f:16075)

[KL] G.R. Krause and T.H. Lenagan, Growth of algebras and Gelfand-Kirillov dimension, revised edition, Graduate Studies in Mathematics, 22, AMS, Providence, RI, 2000. MR.1721834 (2000j:16035)

[LWZ] D.-M. Lu, Q.-S. Wu and J.J. Zhang, Homological integral of Hopf algebras, Trans. Amer. Math. Soc. 359 (2007), no. 10, 4945-4975. MR2320655 (2008f:16083)

[MR] J. C. McConnell and J. C . Robson, Noncommutative Noetherian Rings, Wiley, Chichester, 1987. MR934572 (89j:16023)

[Mo] S. Montgomery, Hopf Algebras and their Actions on Rings, CBMS Regional Conference Series in Mathematics, 82, Providence, RI, 1993. MR.1243637 (94i:16019) 
[SSW] L. W. Small, J.T. Stafford and R. B. Warfield, Jr., Affine algebras of Gelfand-Kirillov dimension one are PI, Math. Proc. Cambridge Philos. Soc. 97 (1985), no. 3, 407-414. MR:778674 (86g:16025)

[WuZ1] Q.-S. Wu and J.J. Zhang, Noetherian PI Hopf algebras are Gorenstein, Trans. Amer. Math. Soc. 355 (2003), no. 3, 1043-1066. MR.1938745 (2003m:16056)

[WuZ2] _ Regularity of involutary PI Hopf algebras, J. Algebra 256 (2002), no. 2, 599-610. MR.1939124 (2004g:16042)

[Zhu] G. Zhuang, Existence of Hopf subalgebras of GK-dimension two, J. Pure Appl. Algebra 215 (2011), 2912-2922. MR 2811574

School of Mathematical Sciences, Qufu Normal University, Qufu, Shandong 273165, People's Republic of China

E-mail address: dgwang@mail.qfnu.edu.cn, dingguo95@126.com

Department of Mathematics, University of Washington, Box 354350, Seattle, WashINGTON 98195

E-mail address: zhang@math.washington.edu

Department of Mathematics, University of Washington, Box 354350, Seattle, WashINGTON 98195

E-mail address: gzhuang@math.washington.edu 